\title{
Top-Hat Representation of Turbulence Statistics in Cloud-Topped Boundary Layers: A Large Eddy Simulation Study
}

\author{
SHOUPING WANG \\ Universities Space Research Association, Huntsville, Alabama \\ BJORn STEVENS \\ Advanced Study Program, National Center for Atmospheric Research, Boulder, Colorado
}

(Manuscript received 14 May 1998, in final form 5 May 1999)

\begin{abstract}
Large eddy simulation is used to study top-hat parameterizations of second- and third-order scalar statistics in cumulus and stratocumulus cloud-topped boundary layers (CTBLs). Although the top-hat parameterizations based on commonly used conditional sampling methods are a useful approach to modeling the vertical fluxes in the simulated CTBLs, they fail to realistically represent the scalar variances. The reason is that the common sampling methods are based at least in part on the sign of vertical velocity, but not on the sign of the scalars whose variances are represented and that scalars and velocity are not perfectly correlated. Furthermore, the self-correlation nature for a variance means that all the fluctuations contribute to its value, while the top-hat models completely ignore the deviations from the top-hat means and thus considerably degrade the representation of the variance. For the fluxes, however, only the coherent convective elements make the most contribution. Analysis of analytic models and "toy" time series indicates in a more generic setting that the effect of poor correlations between the signal upon which the sampling is based and the signal whose variance is to be represented tends to degrade the ability of top-hat parameterizations to capture the variance. The analysis of toy time series also indicates that variability among individual events within a composite degrades the top-hat representation of the variance more than variability within events. For the vertical velocity-scalarrelated third-order moments, the top-hat model gives reasonable estimates for the cumulus CTBL but not for the stratocumulus CTBL. These differences are explained by structural differences (tied to circulation differences in the two CTBLs) in their respective joint probability density functions of vertical velocity and various scalars.
\end{abstract}

\section{Introduction}

It has been a common practice to use the convective mass flux method to represent vertical turbulent fluxes of temperature and moisture in cumulus cloud-topped boundary layers (CTBLs). That is, the fluxes are parameterized as the products of a mass flux and differences between characteristics of convective updrafts and the mean state (e.g., Arakawa and Schubert 1974; Betts 1973). This approach is based on the belief that coherent structures (usually clouds) are responsible for most of the turbulent transport and that these structures are relatively uniform. Because the mass flux method ignores the variability either among or within coherent elements (i.e, updrafts or downdrafts) and only considers the mean properties of some average coherent element, it

Corresponding author address: Shouping Wang, Universities Space Research Association, Global Hydrology and Climate Center, 977 Explorer Blvd., Huntsville, AL 35806.

E-mail: wangsx@vmcs.msfc.nasa.gov may also be referred to as "top-hat" representation of the turbulent fluxes. Recently, Siebesma and Cuijpers (1995) gave excellent discussions on some issues related to the top-hat parameterizations for the trade wind cumulus boundary layers.

The mass-flux decomposition of the flow has also been applied to stratocumulus CTBLs for both observational studies (e.g., Penc and Albrecht 1986; de Laat and Duynkerke 1998; and others) and modeling studies (e.g., Wang and Albrecht 1986; Randall et al. 1992). One critical issue is the construction of a simple and yet physically based conditional sampling that can effectively identify the coherent structures for the top-hat parameterization of the turbulent fluxes. Both Siebesma and Cuijpers (1995) and Schumann and Moeng (1991) discussed this issue.

Recently the top-hat approach has been extended as a means of representing moments other than fluxes. For instance, Randall et al. (1992) used the top-hat formulation to represent the variance of a generic scalar variable $\varphi$ : 


$$
\begin{aligned}
\overline{\varphi^{\prime 2}} & =\alpha_{u}\left(\varphi_{u}-\bar{\varphi}\right)^{2}+\left(1-\alpha_{u}\right)\left(\varphi_{d}-\bar{\varphi}\right)^{2} \\
& =\alpha_{u}\left(1-\alpha_{u}\right)\left(\varphi_{u}-\varphi_{d}\right)^{2},
\end{aligned}
$$

where $\alpha_{u}$ is the fractional coverage of updrafts; $\varphi_{u}$ and $\varphi_{d}$, respectively, represent the value of $\varphi$ averaged over the updrafts and downdrafts; the overbar operator, $(\cdot)$, implies an ensemble averaged value of a variable; and the prime denotes the departure from that average, that is, $\varphi^{\prime}=\varphi-\bar{\varphi}$. They also proposed a similar top-hat parameterization for the flux of the scalar variance:

$$
\begin{aligned}
\overline{w^{\prime} \varphi^{\prime 2}}= & \alpha_{u}\left(w_{u}-\bar{w}\right)\left(\varphi_{u}-\bar{\varphi}\right)^{2} \\
& +\left(1-\alpha_{u}\right)\left(w_{d}-\bar{w}\right)\left(\varphi_{d}-\bar{\varphi}\right)^{2},
\end{aligned}
$$

where $w$ is vertical velocity.

The application of (1) and (2) is appealing for both cloud parameterization and turbulence closure modeling. If one can use (1) to calculate $\overline{q_{t}^{\prime 2}}, \overline{\theta_{l}^{\prime 2}}$, and $\overline{q_{t}^{\prime} \theta_{l}^{\prime}}$ (here, $q_{t}$ is total water mixing ratio and $\theta_{l}$ is liquid water potential temperature) in cumulus CTBLs, then one can use the cloud parameterization of Sommeria and Deardorff (1977) to define cloud cover and liquid water content in a large-scale model. Moreover, (1) and (2) can be used as a closure technique in ensemble turbulence closure models (Randall et al. 1992; Abdella and Mcfarlane 1997). However, Young (1988) found that the tophat model fails to capture the virtual potential temperature variance in a clear convective boundary layer. The applicability of (1) and (2) in the CTBL has, to our knowledge, not been previously studied.

In this work, the large eddy simulation (LES) technique is used to evaluate the top-hat representation for the scalar variance and covariance for both cumulus and stratocumulus CTBLs; we explain the results with discrete joint frequency distribution functions; and we briefly discuss the results of the third moments. Last, we discuss the results from the LES in the context of analytic models and "toy" time series with desirable spectral properties.

\section{LES model}

The LES model used in this study is that of Stevens et al. (1996). It is continually under development, and readers are referred to Stevens et al. (1999) for a complete review and comprehensive evaluation of the current implementation of the model. Of relevance to this study is the fact that monotone operators are used for scalar advection (following Zalesak 1979), the Smagorinsky-Lilly subgrid model with the Smagorinsky constant $C_{s}=0.23$, and a bulk cloud scheme in which liquid water is diagnosed at its equilibrium value. The subgrid-scale (SGS) scalar variances are also calculated (only for diagnostic purpose) from

$$
\left\langle\varphi^{\prime \prime 2}\right\rangle=\frac{2}{C_{h} C_{\varphi}} \frac{\sum_{i=1}^{3}\left\langle u_{i}^{\prime \prime} \varphi^{\prime \prime}\right\rangle^{2}}{\mathrm{TKE}_{s}}
$$

where double primes denote SGS fluctuations, $\langle\cdot\rangle$ a grid volume average, $u_{i}$ velocity component, $\mathrm{TKE}_{s}$ is a diagnosed SGS turbulent kinetic energy, $C_{h}=0.42$, and $C_{\varphi}=1.2$.

The LES code has been used to simulate various cases of CTBLs in the previous five Global Energy and Water Experiment Cloud System Study workshops, and the performance of the model has compared favorably with both other LES models and observations (e.g., Moeng et al. 1996; Bretherton et al. 1999).

For the cumulus CTBL simulation, the LES model uses $100 \times 100 \times 76$ grid points with uniform grid spacing in three dimensions to cover a $6.5 \mathrm{~km} \times 6.5$ $\mathrm{km} \times 3 \mathrm{~km}$ domain, implying $\Delta x=\Delta y=65 \mathrm{~m}$ and $\Delta z=40 \mathrm{~m}$. For the stratocumulus CTBL simulation, the model uses $68 \times 68$ grid points with uniform spacing $\Delta x=\Delta y=50 \mathrm{~m}$ in the horizontal; and there are 76 grid points in the vertical with a minimum spacing of $5 \mathrm{~m}$ within the inversion and $25 \mathrm{~m}$ below the inversion to span a $3.4 \mathrm{~km} \times 3.4 \mathrm{~km} \times 1.97 \mathrm{~km}$ domain. The time step is $2 \mathrm{~s}$ for both simulations.

\section{Cases of shallow cumulus and stratocumulus simulations}

For the shallow cumulus case, we use Barbados Oceanographic and Meteorological Experiment (BOMEX) conditions, which were used in the fifth intercomparison case of the working group 1, held in Seattle, Washington, in July of 1997. This case has a complete set of large-scale conditions and has been studied in detail (Siebesma and Cuijpers 1995). The initial and large-scale conditions can be found in their paper or on the Web site $^{1}$ for the workshop.

For the stratocumulus case we use the San Nicolas Island sounding composited by Albrecht et al. (1995) from observations taken during First International Satellite Cloud Climatology Project (ISCCP) Regional Experiment (FIRE). This sounding represents a classic situation corresponding to persistent stratocumulus. Also, we specify a fixed SST of $288.8 \mathrm{~K}$ and a fixed largescale divergence of $6 \times 10^{-6} \mathrm{~s}^{-1}$. For this case, the fourstream radiation parameterization developed by $\mathrm{Fu}$ and Liou (1992) and Fu et al. (1995) was used to interactively calculate longwave radiative cooling rate.

For both cases the LES is integrated until a quasisteady state is achieved ( $7 \mathrm{~h}$ for the BOMEX case and $4 \mathrm{~h}$ for the FIRE case), and the analysis is conducted over the last hour. The focus is on the top-hat representation of turbulence statistics; thus, we will not discuss the general physical processes associated with the mean and turbulent structures of the simulations, except to say that they are in accord with our expectations and past experience. Nonetheless, for the sake of reference

\footnotetext{
${ }^{1}$ Online at http://www.knmi.nl/ siebesma/bomex.html.
} 

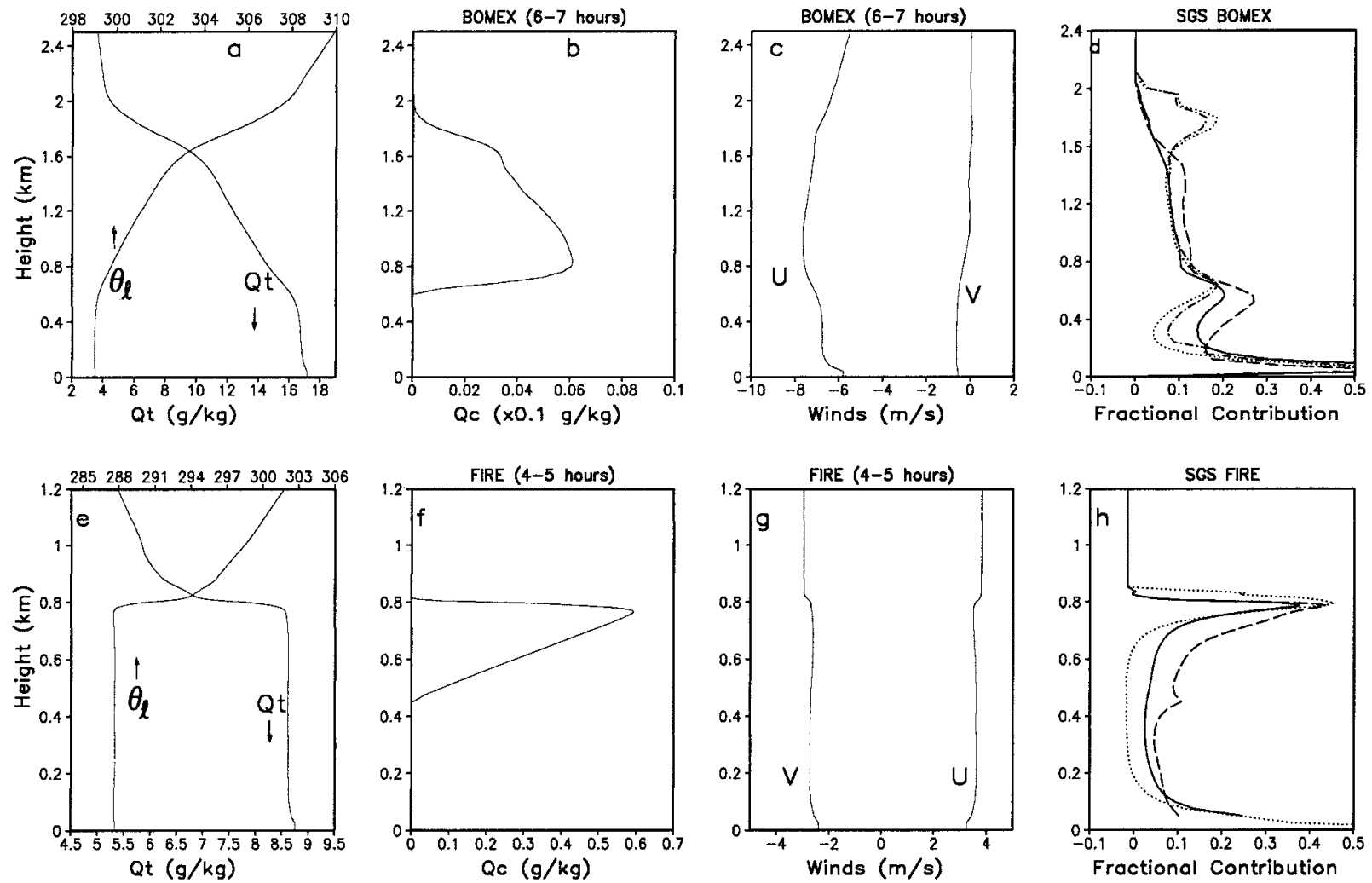

FIG. 1. Mean profiles and SGS statistics from both BOMEX and FIRE simulations. Upper panels are for the BOMEX case, lower panels for the FIRE case. (left to right) Total water mixing ratio $\left(\mathrm{g} \mathrm{kg}^{-1}\right)$ and liquid water potential temperature (K); liquid water content; wind components $u$ and $v\left(\mathrm{~m} \mathrm{~s}^{-1}\right)$; and SGS fractional contributions. For the SGS panels, dotted line denotes the fractional contribution of SGS $q_{t}$ flux; dash-dotted the $\theta_{l}$ flux; solid the $q_{t}$ variance; and long-dashed the $\theta_{l}$ variance.

we have included in Fig. 1 the mean profiles of the simulations averaged over the last hour. As shown in Figs. 1d and 1h, the fractional contributions of the SGS statistics are relatively minor: for the BOMEX case, all the variances and fluxes contribute less than $10 \%$ in the cloud layer; for the FIRE case, the SGS contributions for most of the variables are less than $10 \%$ in the mixed layer, except for $\overline{\left\langle\theta^{\prime \prime 2}\right\rangle}$, which is more than $20 \%$ above $700 \mathrm{~m}$. We emphasize that our study focuses on the regions where the flows are mostly resolvable, that is, the cloud layer for the BOMEX case and the mixed layer below $700 \mathrm{~m}$ for the FIRE case. All the turbulence statistics presented hereafter are for the resolved-scale variables only.

\section{Contributions of top-hat and subplume variability to second moments}

Here we first formulate the decomposition of the variance abstractly; the results from the two case simulations are presented subsequently.

\section{a. Top-hat model}

Consider two variables $a$ and $b$ defined over a set $E$ of $N$ points. One may partition $E$ into $M$ disjoint subsets
$E_{i}$, of length $N_{i}, i=1, \ldots, M$. One can then write the covariance of $a$ and $b$ over $E$, solely in terms of the values of $a$ and $b$ averaged over $E$ and their values defined relative to the $E_{i} \mathrm{~s}$ :

$$
\overline{a^{\prime} b^{\prime}}=\sum_{i=1}^{M} \alpha_{i}\left(a_{i}-\bar{a}\right)\left(b_{i}-\bar{b}\right)+\sum_{i=1}^{M} \alpha_{i}{\overline{a_{i}^{\prime} b_{i}^{\prime}}}^{i},
$$

where the unannotated overbar still represents an average over the set $E$, while the overbar annotated by $i$ represents an average over the $E_{i}$ th subset, that is,

$$
a_{i} \equiv \bar{a}^{i} \equiv \frac{1}{N_{i}}\left(\sum_{E_{i}} a\right)
$$

(similarly for $b$ ). Primes denote deviations from a subset average, so, for instance, $a_{i}^{\prime}=a-a_{i}$. From (4b) it follows that

$$
\bar{a}=\sum_{i=1}^{M} \alpha_{i} a_{i},
$$

where $\alpha_{1}=N_{i} / N$. Because $E$ is a disjoint union of the $E_{i} \mathrm{~s}, \alpha_{1}+\alpha_{2}+\cdots+\alpha_{M}=1$.

The two terms on the rhs of (4a) can be interpreted as follows. The first summation on the rhs of (4a) represents the top-hat contributions, namely, the contri- 
bution to the covariance from the values of $a$ and $b$ composited over each subset $E_{i}$. The second summation in (4a) measures the total contribution from variability within each subset. If a subset is loosely termed plume (analogous to the commonly used updraft and downdraft decomposition), the second summation can be called the subplume variability term. Then, if one can define a physically meaningful conditional sampling method to determine the subsets in such a way that the second summation in (4a) is significantly smaller than the first one, then the covariance can be represented by the first summation. For obvious reasons, this decomposition is most appealing when $M$, the number of subsets, is small.

The above equations are not complete until the $E_{i}$ 's are specified. In the present study, we follow the core decomposition method of Siebesma and Cuijpers (1995) for the BOMEX case, and the $w$-plume approach with zero threshold of Schumann and Moeng (1991) for the FIRE case. These two conditional sampling (CS) methods, denoted by $\mathrm{CS}_{\mathrm{A}}$ (for the BOMEX case) and $\mathrm{CS}_{\mathrm{B}}$ (for the FIRE case), respectively, decompose the flow into two subsets. Thus the second subset $E_{2}$, is simply defined as the set of all points not in $E_{1}$, where the $E_{1}$ 's are defined as follows:

$$
\begin{gathered}
\mathrm{CS}_{\mathrm{A}}: \quad A \text { point } P=(x, y, z, t) \in E_{1} \text { if and only if } \\
w^{\prime}(x, y, z, t)>0, \quad q_{c}(x, y, z, t)>0 \\
\text { and } \theta_{v}(x, y, z, t)-\overline{\theta_{v}}(z, t)>0 ; \\
\mathrm{CS}_{\mathrm{B}}: \quad A \text { point } P=(x, y, z, t) \in E_{1} \text { if and only if } \\
w^{\prime}(x, y, z, t)>0 .
\end{gathered}
$$

In these definitions, $q_{c}$ is liquid water content, and $\theta_{v}$ virtual potential temperature. The subset $E_{2}$ or plume 2 in $\mathrm{CS}_{\mathrm{A}}$ represents the surrounding area of the convective updraft (subset $E_{1}$ ) in the cumulus CTBL and thus will be referred to as the convective environment or simply the environment.

\section{b. Results}

Figure 2 compares the top-hat contributions with the subplume variability for $q_{t}, \theta_{l}, w$ variances, and $q_{t}-$ $\theta_{l}$ covariance from the BOMEX simulation. Clearly, all the variance and covariances are dominated by the environmental subplume variability terms; the contributions from the top-hat terms of the convective updrafts are only $25 \%-30 \%$ of the total values. All the top-hat terms vanish at the cloud-top level. The other two terms in (4a), the convective updraft subplume variability and the environment top-hat terms, contribute little and are neglected. The convective updrafts (subset $E_{1}$ ) occupy only about $2.5 \%$ of the domain. This fractional coverage of the active updraft is close to the value of 3.0\%, typically observed in the trade wind cumulus boundary layers (Albrecht 1981).

We also applied the other two conditional sampling methods discussed by Siebesma and Cujpers (1995). One defines convective updrafts $\left(E_{1}\right)$ solely based on clouds $\left(q_{c}>0\right)$; the other, based on both updrafts and clouds $\left(w^{\prime}>0\right.$ and $\left.q_{c}>0\right)$. These two CS definitions lead to modest increases of updraft fractions to $4 \%$ and $5 \%$ and increases in the top-hat fraction of the scalar variances to $35 \%-40 \%$ (the figures are not shown here).

For the FIRE case, the subplume variability contributions are dominant terms, and the fractional top-hat contributions are only $5 \%-15 \%$ of $\overline{\theta_{l}^{\prime 2}}$ and $20 \%-30 \%$ of $\overline{q_{t}^{\prime 2}}$, as shown in Figs. 3a,b. The covariance $\overline{q_{t}^{\prime} \theta_{l}^{\prime}}$ is small and changes sign in the interior of the boundary layer, a feature that is difficult to model. The top-hat contribution has the wrong sign for the covariance for most of the vertical domain. However, for $\overline{w^{\prime 2}}$, the tophat contribution is greater than the subplume contributions and explains more than $60 \%$ of the total variance, which is consistent with the result of Schumann and Moeng (1991). Although the top-hat model can explain most of the $w$ variance in the FIRE case, it carries only $20 \%$ of the variance for the BOMEX case.

The results for $q_{t}$ and $\theta_{l}$ fluxes are plotted in Fig. 4 for comparison with the scalar variance and covariances. Despite the failure to capture the variance statistics, the same top-hat models well represent the fluxes in the BOMEX case and also give a useful estimate in the FIRE case, in support of the previous studies (Siebesma and Cuijpers 1995; Schumann and Moeng 1991).

\section{Discrete joint frequency distribution function}

To understand why the top-hat model is so successful in representing the fluxes but so poorly represents scalar variances, we find it useful to study the discrete joint frequency distribution function (FDF). If we consider the values of $a^{\prime}$ and $b^{\prime}$ to be random variables $X^{\prime}$ and $Y^{\prime}$, then the FDF is defined as

$$
\begin{array}{r}
f\left(a^{\prime}, b^{\prime}\right)=P\left(a^{\prime}-\Delta a^{\prime} / 2<X^{\prime} \leq a^{\prime}+\Delta a^{\prime} / 2,\right. \\
\left.b^{\prime}-\Delta b^{\prime} / 2<Y^{\prime} \leq b^{\prime}+\Delta b^{\prime} / 2\right),
\end{array}
$$

where $P(A)$ represents the probability that $A$ occurs. The FDF is related to probability density function (PDF) by

$f\left(a^{\prime}, b^{\prime}\right)=\int_{a^{\prime}-\Delta a^{\prime} / 2}^{a^{\prime}+\Delta a^{\prime} / 2} \int_{b^{\prime}-\Delta b^{\prime} / 2}^{b^{\prime}+\Delta b^{\prime} / 2} p d f\left(a^{\prime}, b^{\prime}\right) d b^{\prime} d a^{\prime}$.

Clearly, all the discussions based on FDFs can be easily extended to the PDFs. Because the conditional sampling methods are defined mainly in terms of $w^{\prime}$, we focus on the FDFs for $w^{\prime}$ and scalar fluctuations.

\section{a. BOMEX case}

For the BOMEX case, the relevant features of FDFs do not vary with height within the cloud layer. Thus we discuss these functions only at $1 \mathrm{~km}$. Figures $5 \mathrm{a}-\mathrm{c}$ show the distribution functions $f\left(w^{\prime}, \varphi^{\prime}\right)$, where $\varphi^{\prime} \in\left(q_{t}^{\prime}\right.$, $\left.\theta_{l}^{\prime}, \theta_{v}^{\prime}\right)$. Also displayed in Figs. 5d-f are the scatterplots 

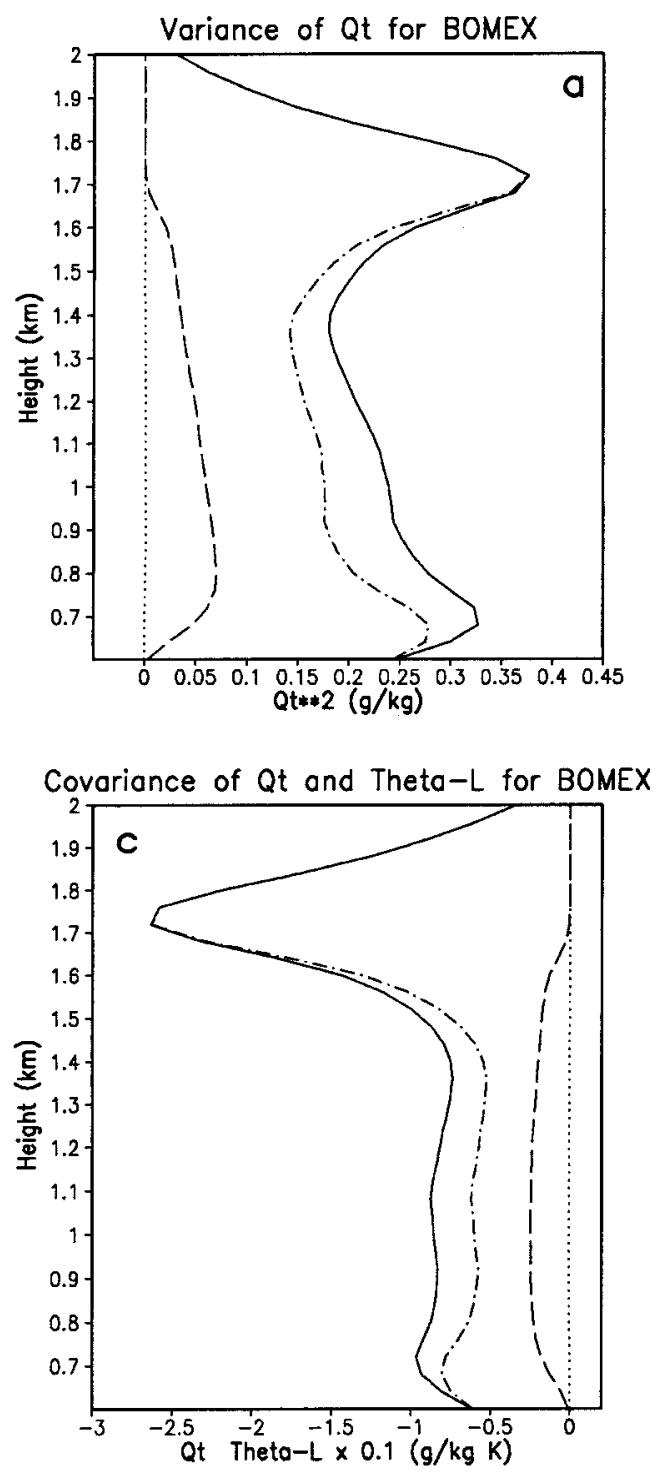
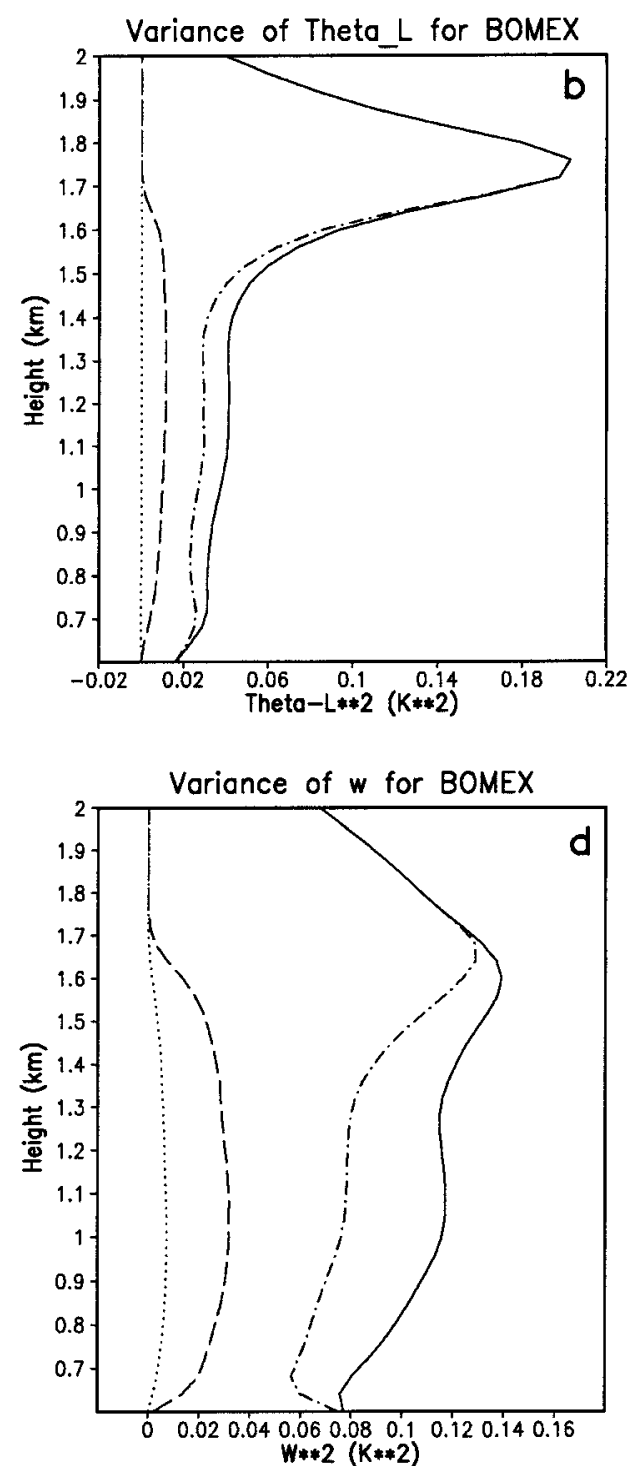

FIG. 2. Contributions of top-hat and subplume variability to various variance and covariance statistics in the BOMEX simulation. (a) $\overline{q_{t}^{\prime 2}}$, (b) $\overline{\theta_{l}^{\prime 2}}$, (c) $\overline{q_{t}^{\prime} \theta_{l}^{\prime}}$, and (d) $\overline{w^{\prime 2}}$. Solid lines denote total values of the statistics; long-dashed, the contribution of convective updraft top-hat terms; dotted, the contribution of the updraft subplume variability; and dashdotted, the contribution of the environment subplume variability.

of $w^{\prime}$ and $\varphi^{\prime}$ sampled at 20 different times for the convective updrafts defined as plume 1 by $\mathrm{CS}_{\mathrm{A}}$. Clearly, the sampled $w^{\prime}$ and $\varphi^{\prime}$ are highly correlated and coincide with the long "tail" areas of $f\left(w^{\prime}, \varphi^{\prime}\right)$.

An important characteristic of these distributions, particularly for $f\left(w^{\prime}, q_{t}^{\prime}\right)$ and $f\left(w^{\prime}, \theta_{l}^{\prime}\right)$, is that in the environment, each one tends to be approximately symmetric about $w^{\prime}=0$ and $\varphi^{\prime}=0$ [i.e., $f\left(w^{\prime}, \varphi^{\prime}\right) \cong$ $f\left(-w^{\prime}, \varphi^{\prime}\right)$ and $\left.f\left(w^{\prime}, \varphi^{\prime}\right) \cong f\left(w^{\prime},-\varphi^{\prime}\right)\right]$. This feature of symmetry implies poor correlation between $w^{\prime}$ and $\varphi^{\prime}$ in the environment. In addition, the fluctuations in the environment are generally significantly weaker than those in the convective updrafts, reflecting the lack of convective activity there. Thus, the environment terms (the plume-2 terms) in (4a), including both top-hat and subplume variability, are very small, and the total flux $\overline{w^{\prime} \varphi^{\prime}}$ can be determined approximately by only the updraft contribution. Furthermore, because the local fluctuations $\varphi_{1}^{\prime}=\varphi-\varphi_{1}$ are, in general, smaller than $\varphi_{1}$ $-\bar{\varphi}$, and because some of the positive and negative values of $w_{1}^{\prime} \varphi_{1}^{\prime}$ are canceled out in (4a), the convective updraft subplume variability makes a very small contribution to the scalar fluxes.

This FDF analysis demonstrates that the success of the top-hat models to represent the fluxes in this case depends on the contrast of the very different correlation functions between $w^{\prime}$ and $\varphi^{\prime}$ in the environment and that in the convective updrafts, or the bimodal behavior 

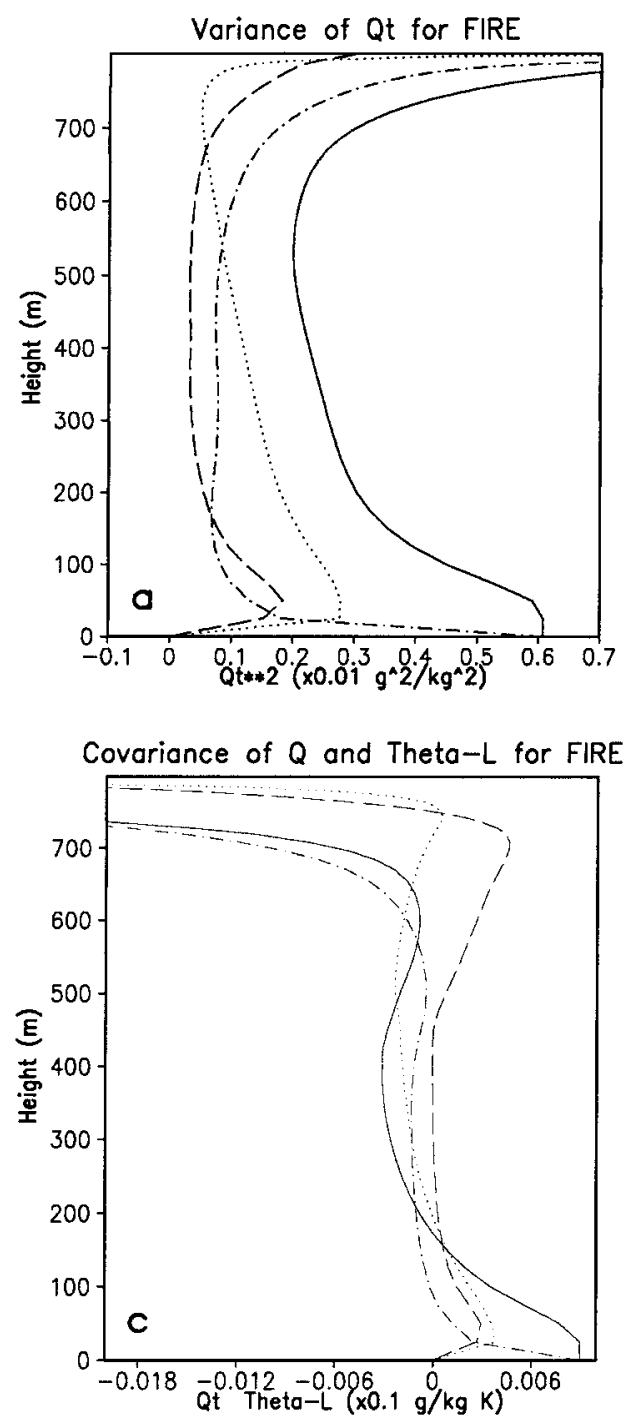
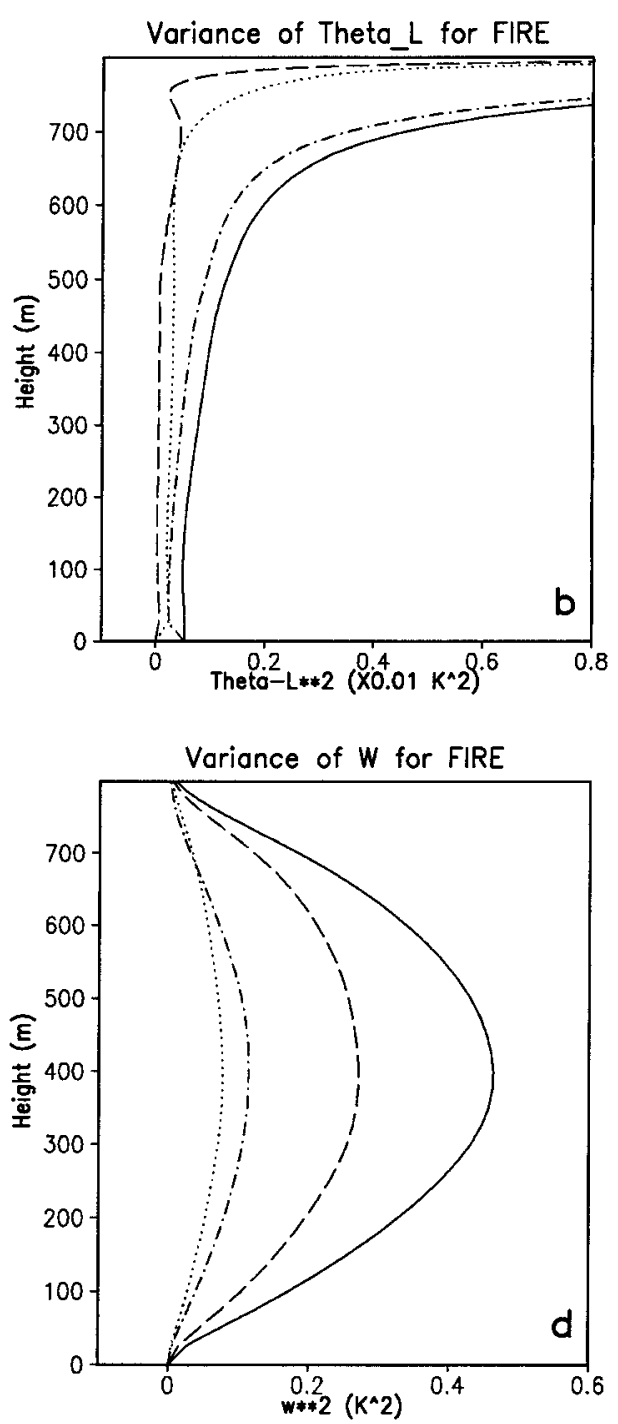

FIG. 3. Contributions of top-hat and subplume variability to various variances and covariances in the FIRE simulation. (a) $\overline{q_{t}^{\prime 2}}$, (b) $\overline{\theta_{l}^{\prime 2}}$, (c) $\overline{q_{t}^{\prime} \theta_{l}^{\prime}}$, and (d) $\overline{w^{\prime 2}}$. Solid lines denote total values of the statistics; long-dashed, the total top-hat contribution; dotted, the contribution of the updraft subplume variability, and dash-dotted, the downdraft subplume variability.

of the FDFs in the two plumes. We notice that in the environment positive and negative values of each of the turbulent fluctuations, including both $w^{\prime}$ and $\varphi^{\prime}$, are almost canceled out due to the broad sampling area (97\% of the domain), leading to the fact that the environmental averaged variables are very close to the ensemble mean. This cancellation of negative and positive values in an individual plume significantly degrades the top-hat representation of some statistics, especially scalar variances for the CTBLs.

As shown in Fig. 6, a significant difference between $f\left(\theta_{l}^{\prime}, q_{t}^{\prime}\right)$ and $f\left(w^{\prime}, \varphi^{\prime}\right)$ is that $q_{t}^{\prime}$ and $\theta_{l}^{\prime}$ are strongly negatively correlated not only in the updrafts where the liquid water fluctuations dominate both $q_{t}^{\prime}$ and $\theta_{l}^{\prime}$, but also in the environment that is influenced by the detrained nonbuoyant air from the updrafts. This strong anticorrelation results in a significant environmental contribution to the total covariance $\overline{q_{t}^{\prime} \theta_{l}^{\prime}}$. However, the environmental top-hat term in (4a), that is, $\left(q_{t_{2}}-\right.$ $\left.\overline{q_{t}}\right)\left(\theta_{l_{2}}-\overline{\theta_{l}}\right)$, is close to zero because the environmental averages are very close to the ensemble mean. Thus, the contributions from the environment based on $\mathrm{CS}_{\mathrm{A}}$ are predominantly accounted for by the subplume variability term. Therefore, the top-hat model fails to represent $\overline{q_{t}^{\prime} \theta_{l}^{\prime}}$ for this case.

The same argument applies to the case of variance. Since any turbulent fluctuation perfectly correlates with itself, the environmental contributions must be included in the parameterization. However, the conditional sampling method for the BOMEX case, $\mathrm{CS}_{\mathrm{A}}$, results only in a trivial contribution from the environmental top-hat terms, due to the cancellation of the negative and pos- 

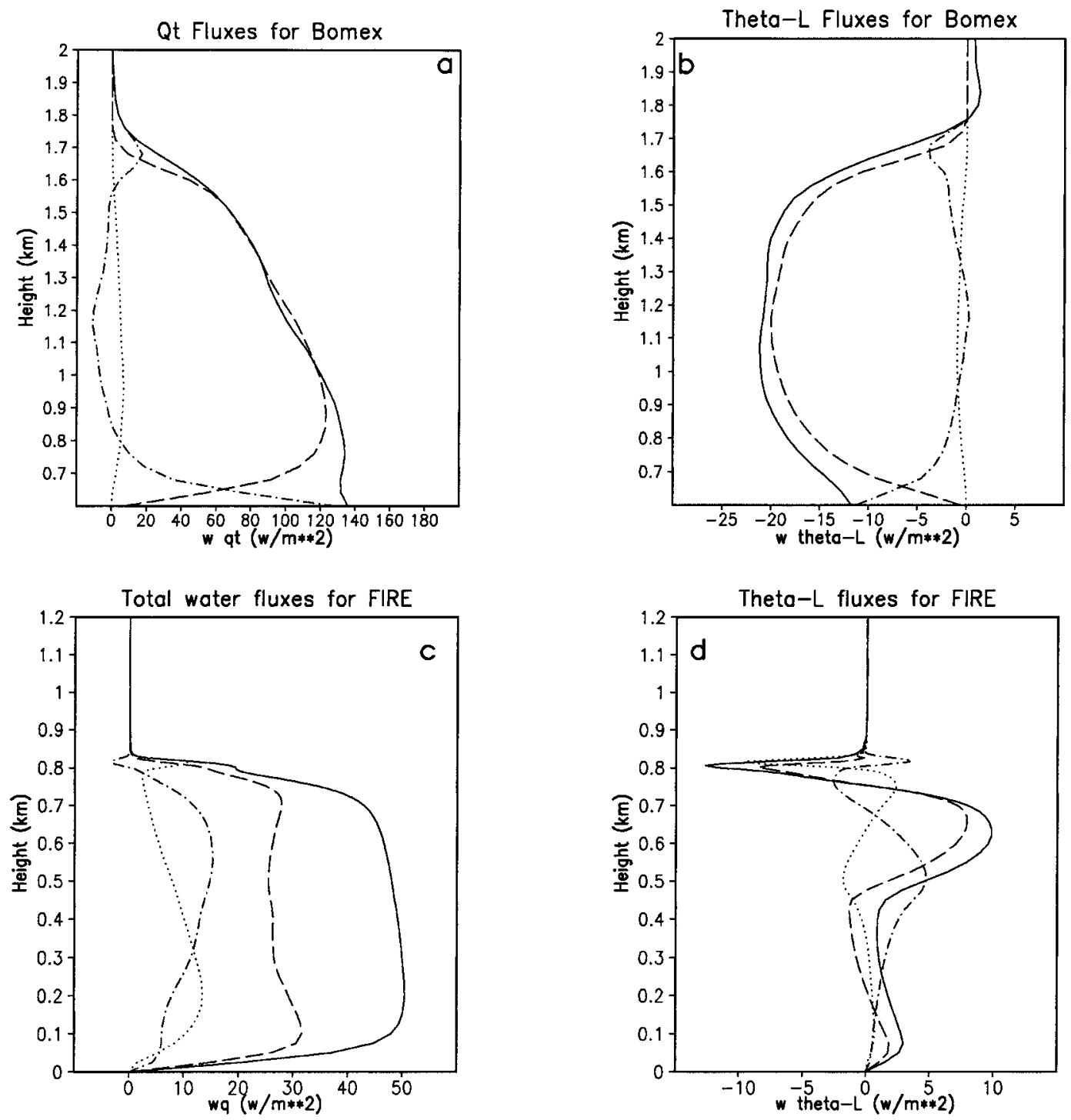

FIG. 4. Contributions of top-hat and subplume variability to the turbulent fluxes in both simulations. (left) $\rho L \overline{w^{\prime} q_{t}^{\prime}}$ and (right) $\rho c_{p} \overline{w^{\prime} \theta_{l}^{\prime}}$. Upper panels are for the BOMEX case and lower panels for the FIRE case. Solid lines denote total values of the fluxes; long-dash, the total top-hat contribution; dotted, the contribution of the updraft subplume variability, and dash-dotted, the environment or downdraft subplume variability.

itive fluctuations. Consequently, the contributions from the environment subplume variability terms dominate the total environmental plume terms. Therefore, the tophat model is not sufficient to capture the variances.

The above examples suggest that the coherent structure may not carry most of the scalar variance and covariances for cumulus CTBLs. Therefore, one should design a conditional sampling method not only to identify the coherent structure, but also to represent the variability in the environment. This requires that the positive definite nature of a variance be preserved in the top-hat model. For example, we define a new conditional sampling method $\mathrm{CS}_{\mathrm{C}}$ by adding one more subset (plume) to the BOMEX conditional sampling method:
$\mathrm{CS}_{\mathrm{C}}$ : $\quad A$ point $P=(x, y, z, t) \in E_{1}$ if and only if

$w^{\prime}(x, y, z, t)>0, \quad q_{c}(x, y, z, t)>0 \quad$ and

$\theta_{v}(x, y, z, t)-\overline{\theta_{v}}(z, t)>0$;

$P \in E_{2}$, if and only if $w^{\prime}(x, y, z, t)>0$ and

$$
P \notin E_{1} ;
$$

$P \in E_{3}$, otherwise.

This conditional sampling method not only defines a convective plume, but also separates upward and downward streams in the environment, each of which occupies a similar fractional coverage. Thus, it guarantees 

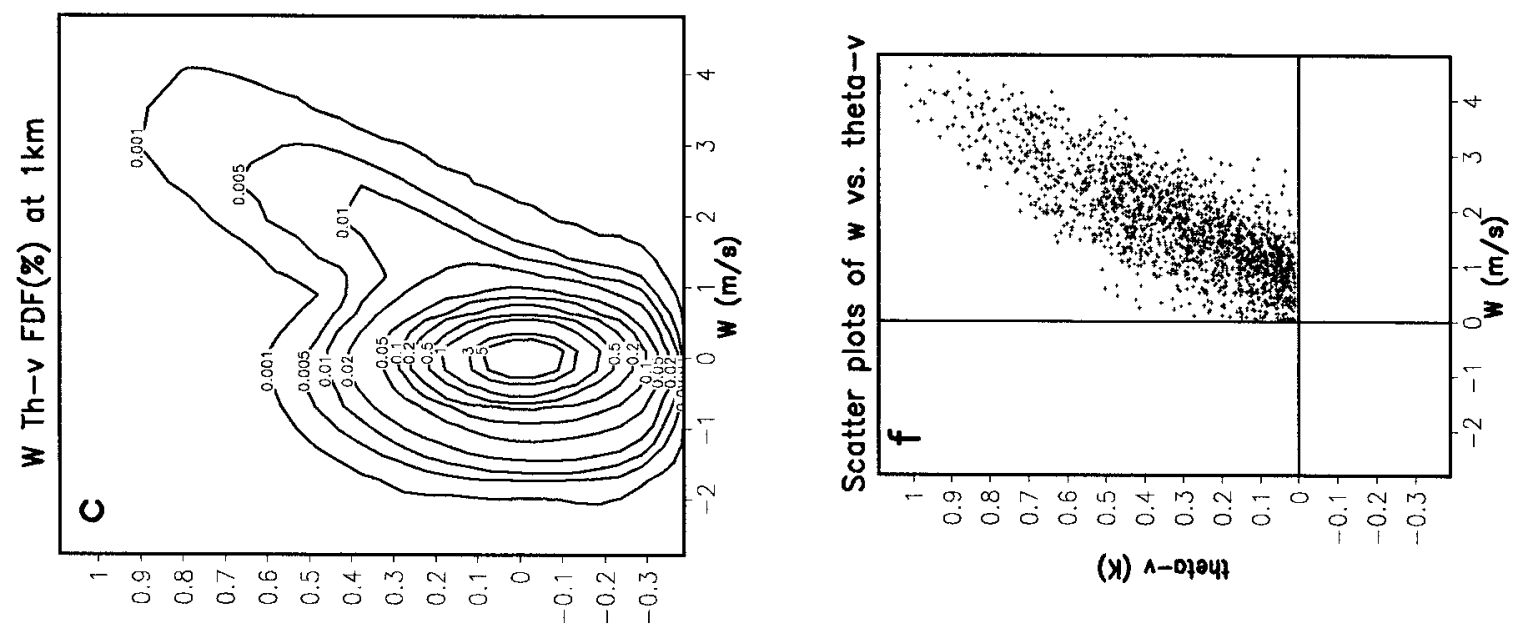

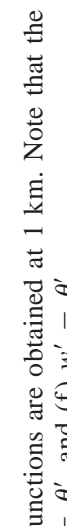

$(x) \wedge-0 j$ 이
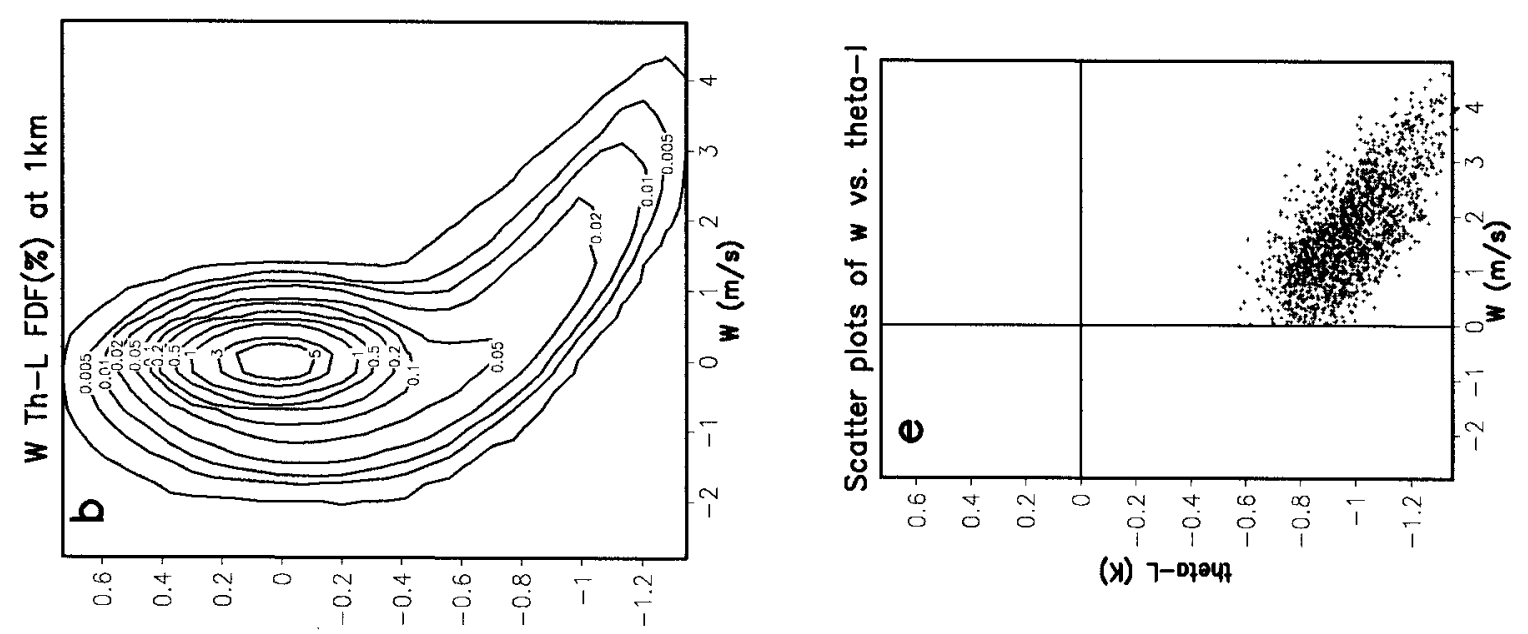

(x) 7-Diaut
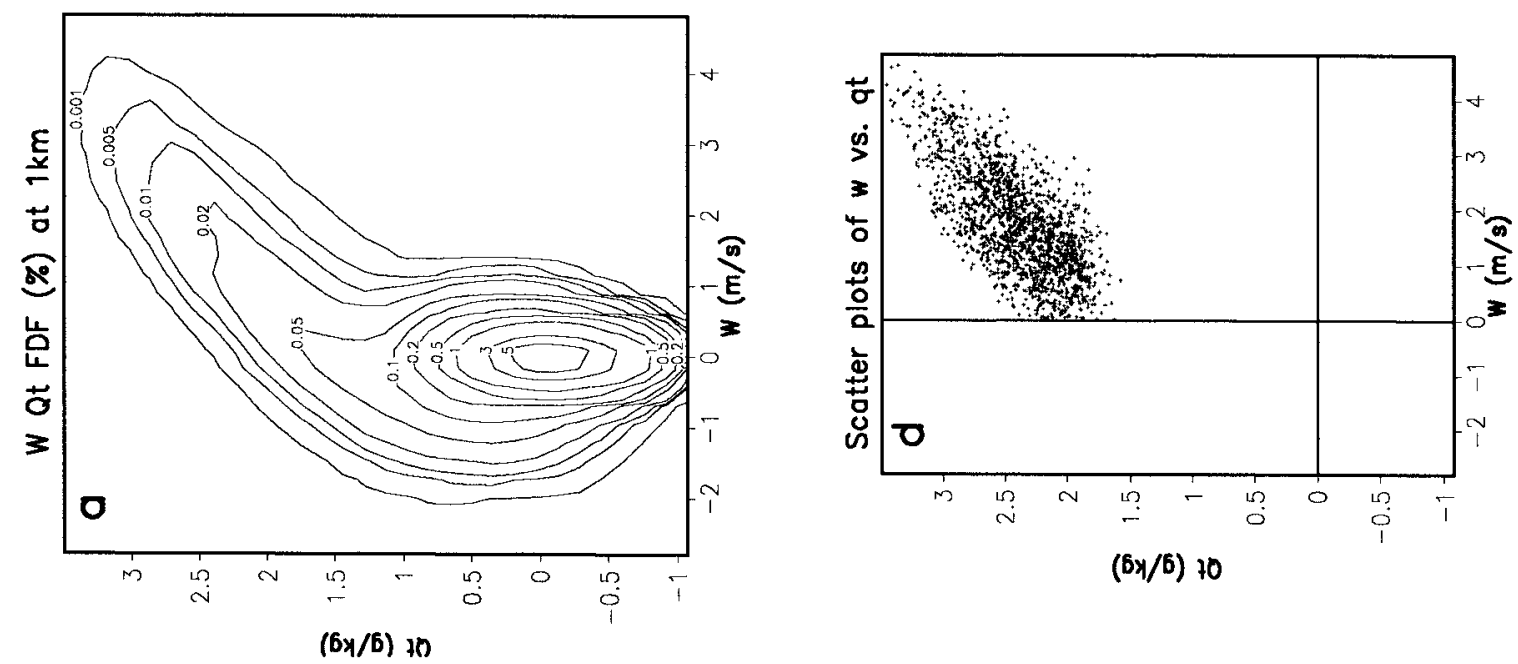

(1)

(x) 7-07047

$(6 x / 6) 70$ 

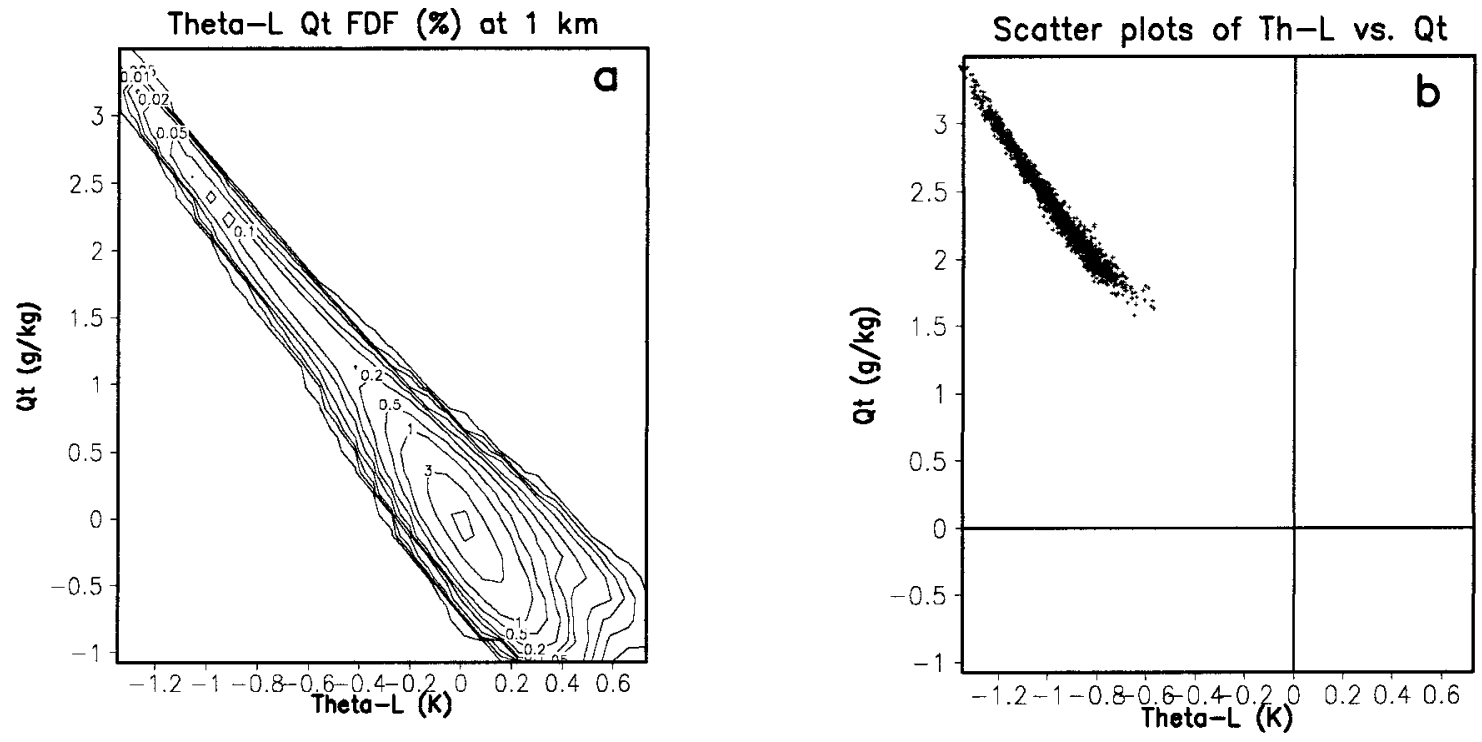

FIG. 6. (a) Joint frequency distribution function and (b) the scatterplots for $\theta_{l}^{\prime}$ and $q_{t}^{\prime}$ of the convective updrafts from the BOMEX simulation.

that $w^{\prime}$ has only one sign in each of these plumes. As shown in Fig.7a, the top-hat terms based on the above conditional sampling can explain $50 \%-60 \%$ of the resolved $\overline{w^{\prime 2}}$ (similar to what was explained for the FIRE case), a fraction significantly larger than that in Fig. 2d, even though it is still well below the top-hat fraction of the fluxes. However, the above sampling approach does not at all improve the top-hat parameterization of $\overline{q_{t}^{\prime 2}}$ as shown in Fig. 7b, since $q_{t}^{\prime}$ and $w^{\prime}$ are poorly correlated in the convective environment.

If we apply $\mathrm{CS}_{\mathrm{B}}$ (in which the updrafts are defined only by $w^{\prime}>0$ ) to the BOMEX case, the top-hat fraction of $w$ variance is reduced to $40 \%$ (figures not shown here) compared to $60 \%$ for $\mathrm{CS}_{\mathrm{C}}$ (Fig. 7a). In addition, the top-hat fluxes of $q_{t}$ and $\theta_{l}$ account for about $40 \%$ of the total fluxes at the cloud base and decrease to only $10 \%$ at $1 \mathrm{~km}$, which is clearly a result of the failure by the $w^{\prime}$-sign-only scheme to identify the coherent structure, that is, cloudy updrafts, in this case. This result is consistent with Siebesma (1996).

\section{b. FIRE case}

We found that the relevant structures and patterns of the various distribution functions are similar within the cloud layer. Therefore, it is sufficient to show these functions at one level in the cloud layer. Figure 8 displays various FDFs at $637.2 \mathrm{~m}$, where $\overline{w^{\prime} \theta_{l}^{\prime}}$ is large. The distribution functions $f\left(w^{\prime}, q_{t}^{\prime}\right)$ and $f\left(w^{\prime}, \theta_{v}^{\prime}\right)$ show positive correlation in general, while $f\left(w^{\prime}, \theta_{l}^{\prime}\right)$ tends to be quasi-symmetric about $\theta_{l}^{\prime}=0$, implying that the variables are poorly correlated.

The lower left quadrants in Figs. 8a,b $\left(w^{\prime}<0 ; q_{t}^{\prime}\right.$ and $\left.\theta_{v}^{\prime}<0\right)$ show that the entrainment is associated with the drier inversion air and the cloud-top radiative and evaporative cooling. These processes tend to make downward parcels negatively buoyant and dry. The upper left quadrant of Fig. 8b shows the entrainment of more buoyant inversion air into the cloud layer. The distribution function $f\left(w^{\prime}, \theta_{l}^{\prime}\right)$ demonstrates a significant variability for $w^{\prime}<0$, as shown in Fig. 8c. The entrainment of warmer air from the inversion and the radiative cooling of saturated parcels are clearly two major processes that simultaneously regulate the distribution. If the radiative cooling dominates, negative $\theta_{l}^{\prime}$ is associated with negative $w^{\prime}$ in the lower left quadrant in the distribution. On the other hand, if the entrainment mixing is dominant, positive $\theta_{l}^{\prime}$ is associated with negative $w^{\prime}$ as shown in the upper left quadrant to increase the negative correlation between $w^{\prime}$ and $\theta_{l}^{\prime}$. Apparently, there is significantly larger variability in $\theta_{l}$ in the downdrafts than in the updrafts, which explains why the contribution from the downdraft variability dominates the total $\theta_{l}$ variance as shown in Fig. 3b. Figure $8 \mathrm{~d}$ shows that the correlation between $q_{t}^{\prime}$ and $\theta_{l}^{\prime}$ is very weak, due to the well-mixed mean profiles. The tendency of negative correlation in the lower right corner, that is, negative $q_{t}^{\prime}$ associated with positive $\theta_{l}^{\prime}$, apparently implies effects of the entrainment.

It is evident that the poor correlation of $\varphi^{\prime}$ with $w^{\prime}$, particularly $\theta_{l}^{\prime}$ with $w^{\prime}$, in each of the plumes considerably reduces the significance of the top-hat contributions to the variances, because the positive and negative scalar fluctuations within each plume are canceled out. Thus, for top-hat representation of a variance, it is important to maintain a unique sign, positive or negative, for turbulent fluctuations in each of the plumes defined by a conditional sampling. For example, to improve the representation of $\overline{q_{t}^{\prime 2}}$, the " $w q$-plume" method 

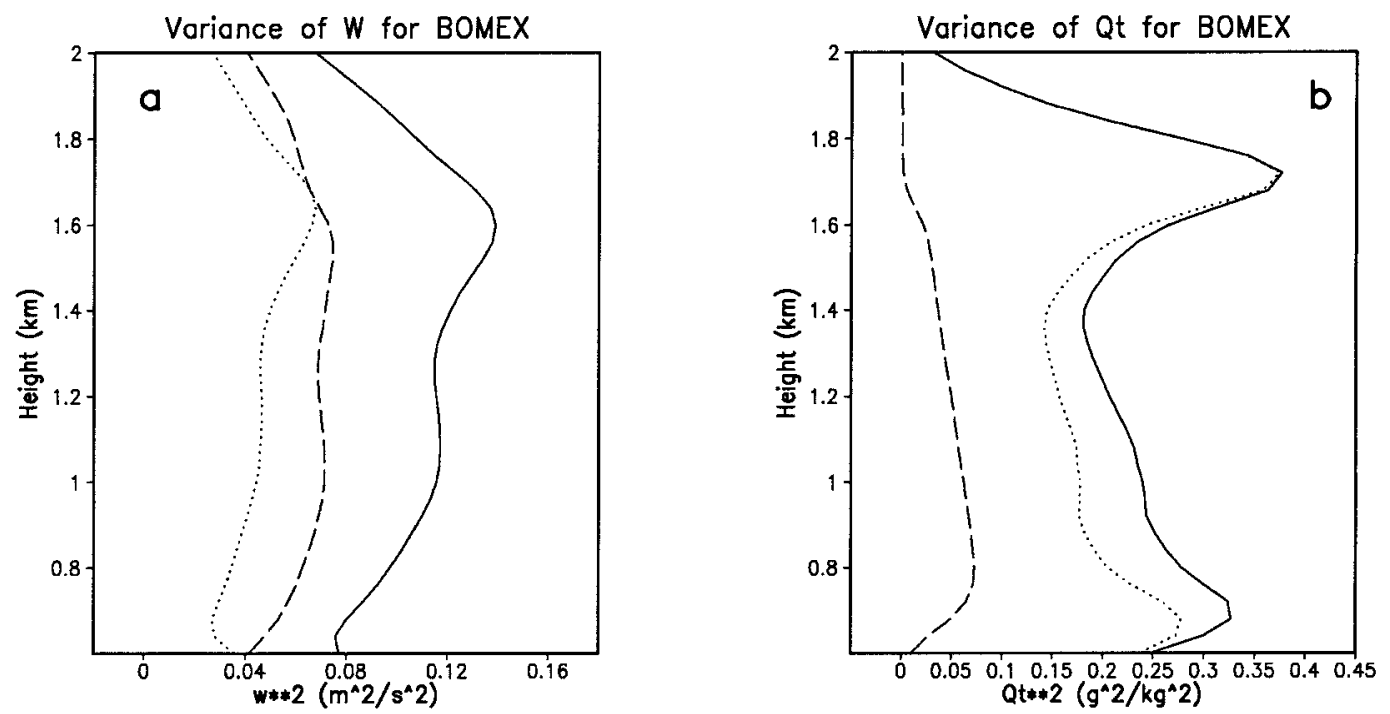

FIG. 7. Contributions of top-hat and subplume variability to $w$ and $q_{t}$ variances based on the $\mathrm{CS}_{\mathrm{C}}$ for the BOMEX case. (a) $\overline{w^{\prime 2}}$ and (b) $\overline{q_{t}^{\prime 2}}$. Solid lines represent total variances; long-dashed, the total top-hat contribution; and dotted, the total subplume variability.

by Schumman and Moeng (1991) is tested here. That is,

$\mathrm{CS}_{\mathrm{D}}: \quad A$ point $P=(x, y, z, t) \in E_{1}$ if and only if

$w^{\prime}(x, y, z, t)>0, \quad$ and $\quad q_{t}^{\prime}(x, y, z, t)>0$

$P \in E_{2}$ if and only if $w^{\prime}(x, y, z, t)<0$ and

$$
q_{t}^{\prime}(x, y, z, t)<0
$$

$P \in E_{3} \quad$ otherwise.

This sampling scheme guarantees that $q_{t}^{\prime}$ keeps a unique sign for plume 1 and 2 , which represent the areas of the upper right and lower left quadrants in Fig. 8a. Figure 9a shows that the top-hat representation using $\mathrm{CS}_{\mathrm{D}}$ contributes significantly more to the total $\overline{q_{t}^{\prime 2}}$ than it does with $\mathrm{CS}_{\mathrm{B}}$ (Fig. 3). However, since $q_{t}^{\prime}$ is poorly correlated with $\theta_{l}^{\prime}$, the uniqueness of the $q_{t}^{\prime}$ sign in the plumes does not lead to the uniqueness of the $\theta_{l}^{\prime}$ sign. Consequently, the new sampling method does not at all improve the top-hat parameterization of $\overline{\theta_{l}^{\prime 2}}$, which is again dominated by the subplume variability, as shown in Fig. 9b. This example demonstrates that the major difficulty of the top-hat parameterization in this case is the poor correlation among $w^{\prime}, \theta_{l}^{\prime}$, and $q_{t}^{\prime}$.

It is now clear why the top-hat parameterizations of the scalar variances are less successful than those of the fluxes. First, in the case of variance, the self-correlation means that all the fluctuations contribute, while the tophat models completely ignore the deviations from the plume mean values. For the fluxes, however, only coherent elements make significant contribution, while the deviations from the top-hat values [second term in (4a)] may cancel out due to poor correlation. Second, the commonly used conditional sampling schemes are based mainly on the vertical velocity and other related variables and thus are less suitable for the scalar variances than for the fluxes, due to the poor correlation between the velocity and the scalars.

\section{The contributions to third moments}

Third moments can also be written as a sum of contributions from plume top-hat and subplume variability:

$$
\begin{aligned}
& \overline{a^{\prime} b^{\prime 2}}=\alpha_{1}\left(a_{1}-\bar{a}\right)\left(b_{1}-\bar{b}\right)^{2} \\
& +\left(1-\alpha_{1}\right)\left(a_{2}-\bar{a}\right)\left(b_{2}-\bar{b}\right)^{2} \\
& +\alpha_{1}\left[{\overline{a_{1}^{\prime} b_{1}^{\prime 2}}}^{1}+2\left(b_{1}-\bar{b}\right){\overline{a_{1}^{\prime} b_{1}^{\prime}}}^{1}+\left(a_{1}-\bar{a}\right){\overline{b_{1}^{\prime 2}}}^{1}\right] \\
& +\left(1-\alpha_{1}\right) \\
& \times\left[{\overline{a_{2}^{\prime} b_{2}^{\prime 2}}}^{2}+2\left(b_{2}-\bar{b}\right){\overline{a_{2}^{\prime} b_{2}^{\prime}}}^{2}+\left(a_{2}-\bar{a}\right){\overline{b_{2}^{\prime 2}}}^{2}\right],
\end{aligned}
$$

where all the notations are the same as in (4a), except that only two subsets (or plumes) are considered here. The first two terms in the rhs of (7) are top-hat terms, and the rest are subplume variability contributions. We only consider $w^{\prime} \varphi^{\prime}$-related third moments.

Because the frequency distribution of $w^{\prime}$ and $\varphi^{\prime}$ in the BOMEX case tends to be approximately symmetric about $w^{\prime}=0$ and $\varphi^{\prime}=0$ in the environment, one can easily show that $\overline{w^{\prime} \varphi^{\prime 2}}$ and $\overline{w^{\prime 2} \varphi^{\prime}}$ are dominated by the convective plume contributions (see appendix A). Therefore, one should be able to use the top-hat model to parameterize them so long as the contributions from the updraft subplume variability are small. This result is presented in Fig. 10. The top-hat model gives a better representation of $\overline{w^{\prime} \varphi^{\prime 2}}$ than $\overline{w^{\prime 2} \varphi^{\prime}}$ because the symmetry about $w^{\prime}=0$ is better than that about $\varphi^{\prime}=0$. 

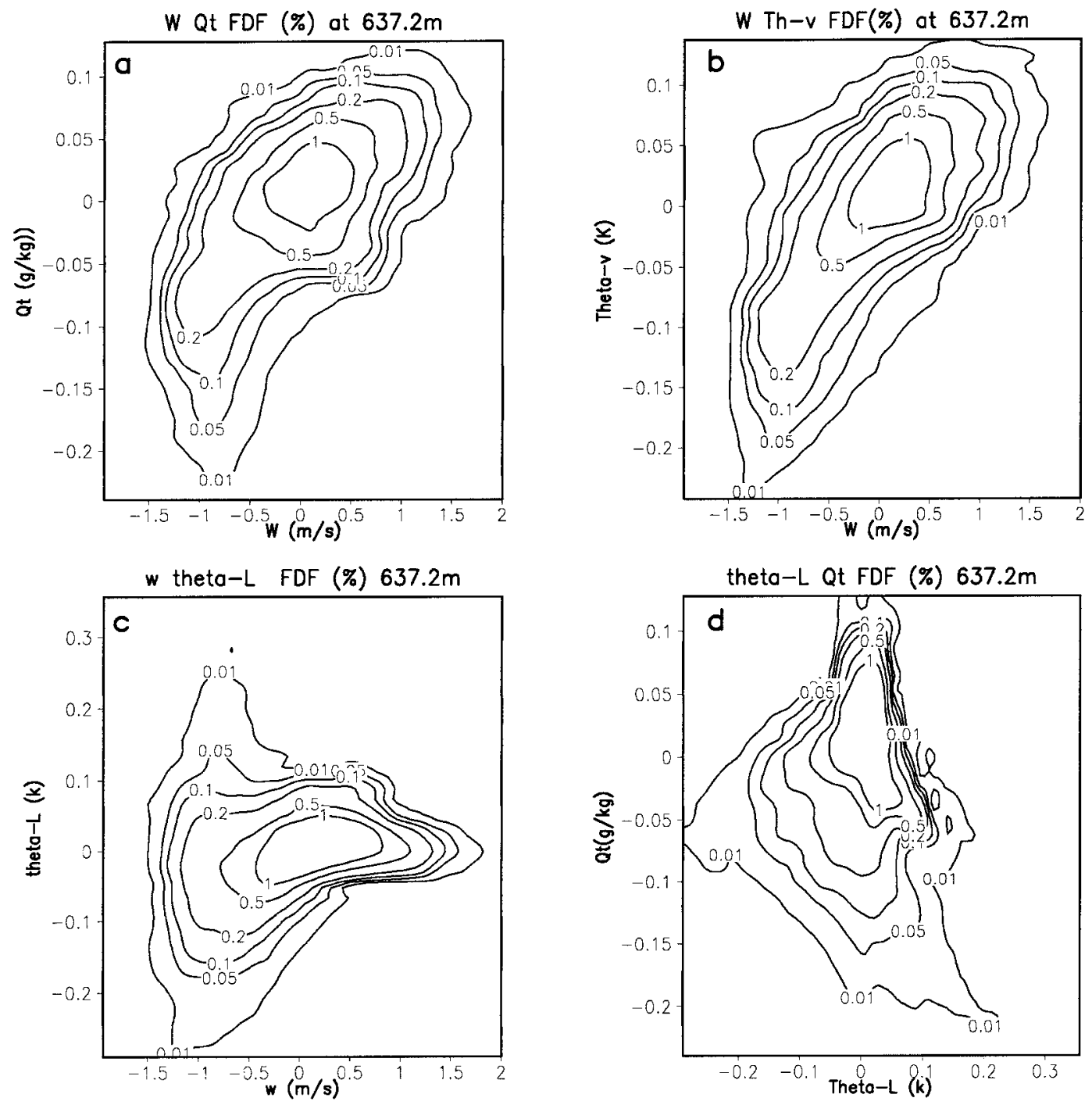

FIG. 8. Various joint frequency distribution functions at $637.2 \mathrm{~m}$ for the FIRE case. (a) $f\left(w^{\prime}, q_{t}^{\prime}\right)$, (b) $f\left(w^{\prime}, \theta_{v}^{\prime}\right),($ c) $f\left(w^{\prime}, \theta_{l}^{\prime}\right)$, and (d) $f\left(\theta_{l}^{\prime}, q_{t}^{\prime}\right)$. Note that the numerical values are in the unit of percentage.

For the FIRE case, the top-hat contributions do not provide useful estimates for any of the four third-order moments, as shown in Fig. 11. In fact, the sum of the top-hat contributions from the updrafts and downdrafts is close to zero for each of these statistics. The reasons are as follows. First, the plume-scale variabilities [i.e., $\left(\varphi_{1}-\bar{\varphi}\right)$ and $\left.\left(\varphi_{2}-\bar{\varphi}\right)\right]$ are small, due to the existence of positive and negative fluctuations in each plume. Second, the plume-scale variability tends to be symmetric, that is, $\left(\varphi_{1}-\bar{\varphi}\right)$ and $\left(\varphi_{2}-\bar{\varphi}\right)$ have similar magnitudes but different signs, so that the first and second terms in (7) are nearly canceled out, leading to the dominance of the subplume variability contributions. Although the top-hat contributions tend to be symmetric, the subplume contributions are highly asymmetric, particularly for $\overline{w^{\prime 2} q_{t}^{\prime}}$ and $\overline{w^{\prime} q_{t}^{\prime 2}}$, as shown in Figs. 11a,b, reflecting the influences of the cloud-top and surface mixing pro- cesses. For $\overline{w^{\prime 2} \theta_{l}^{\prime}}$ and $\overline{w^{\prime} \theta_{l}^{\prime 2}}$, the subplume contribution in downdrafts clearly dominates, which can be explained by the large $\theta_{l}^{\prime}$ variability in downdrafts shown by the FDF $f\left(w^{\prime}, \theta_{l}^{\prime}\right)$ in Fig. 8c.

\section{Discussion}

As illustrated with the two examples above, the failure of the top-hat parameterization to adequately represent scalar variances is in part due to issues of sampling and in part due to the generic variability of the signal. Even with perfect correlations between scalars and $w^{\prime}$, the variability of a scalar within different plumes leads to significant contributions to the variance by the subplume variability in (4a). In this section we make some simple assumptions and look more generically at how different features of a field contribute to the ability 

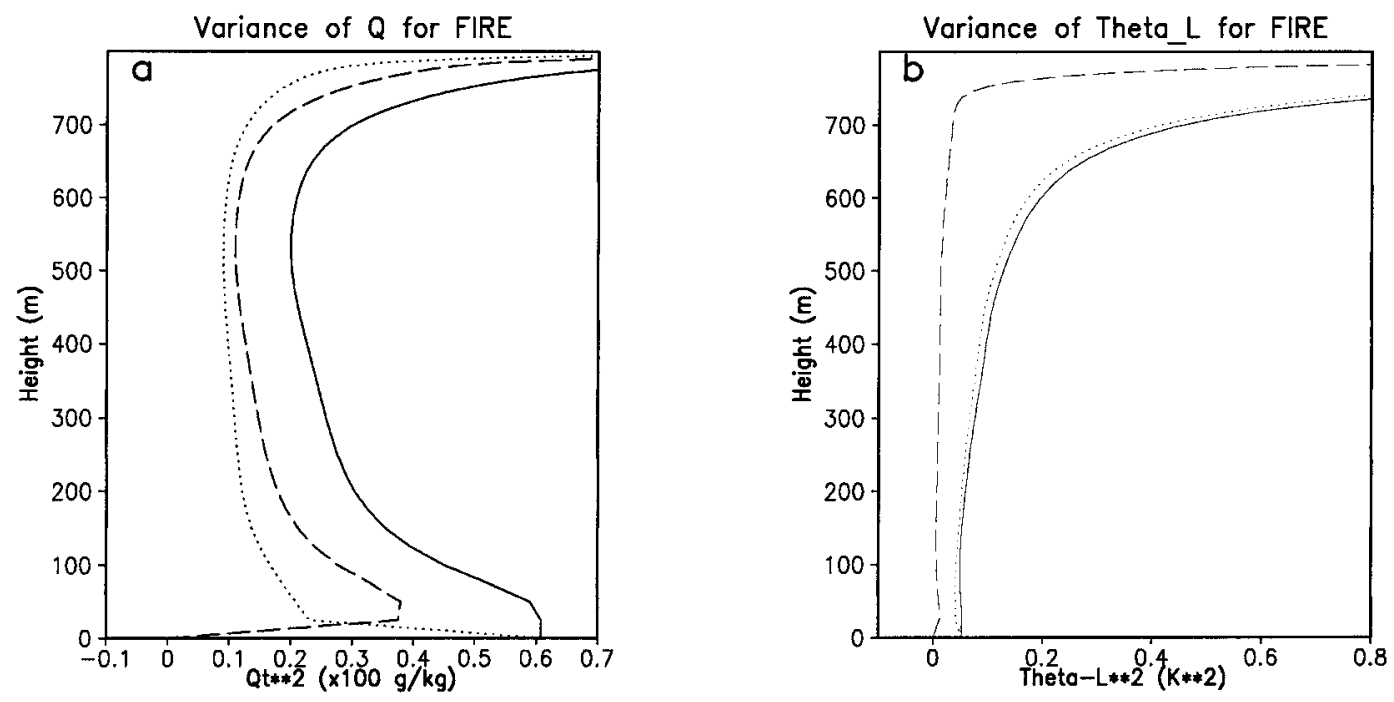

FIG. 9. Contributions of top-hat and subplume variability to $q_{t}$ and $\theta_{l}$ variances based on the $\mathrm{CS}_{\mathrm{D}}$. (a) $q_{t}$ variance and (b) $\theta_{l}$ variance. Solid lines represent total variances; long-dashed, the total top-hat contribution; and dotted, the total subplume variability.

of top-hat parameterizations to represent the variance of that field.

\section{a. Jointly Gaussian processes}

Here we consider the ability of a top-hat parameterization to represent the variance of a scalar $a$, given the decomposition of the field through $\mathrm{CS}_{\mathrm{B}}$, and the assumption that the vertical velocities $w$ and $a$ are jointly distributed by a Gaussian process with correlation coefficient $\rho=\overline{w^{\prime} a^{\prime}} /\left(\sigma_{a} \sigma_{w}\right)$, where now we use $\sigma_{x}^{2}$ to denote the variance of a field $x$. Because we use the sampling method $\mathrm{CS}_{\mathrm{B}}$, we can speak of our sets as the set of updrafts $E_{u}$ and the set of downdrafts $E_{d}$. Because $w$ and $a$ are distributed jointly through a Gaussian process, $E_{u}$ and $E_{d}$ must each compose an equal fraction of the set $E$ (i.e., $\alpha_{u}=\alpha_{d}=1 / 2$ ). Furthermore, $a_{u}$ and $a_{d}$, that is, the value of $a$ averaged over updrafts and downdrafts, respectively, can be calculated analytically (e.g., Wyngaard and Moeng 1992):

$$
a_{d}=\frac{-2 \rho \sigma_{a}}{\sqrt{2 \pi}}, \text { and } a_{u}=\frac{2 \rho \sigma_{a}}{\sqrt{2 \pi}},
$$

which implies that the variance of $a$ can be written as

$$
\sigma_{a}^{2}=\frac{2 \sigma_{a}^{2} \rho^{2}}{\pi}+\mathrm{SPV}
$$

where the first term above is the top-hat part defined by (1), and the subplume variability is denoted by SPV. Thus it follows that

$\mathrm{SPV}=\sigma_{a}^{2}\left(1-\frac{2 \rho^{2}}{\pi}\right) \Rightarrow\left(1-\frac{2}{\pi}\right) \leq \frac{\mathrm{SPV}}{\sigma_{a}^{2}} \leq 1$.

Equation (10) also shows that under the above described conditions the subplume variability is always significant and accounts for between about $40 \%$ and $100 \%$ of the total variance.

Equation (10) illustrates that the correlation between the sampling field (in this case $w$ ) and the field whose variance we wish to know determines in large part the partitioning of the variance between the top-hat and SPV terms. Note that because the correlation is the flux divided by the product of the square root of the variances, it is possible that there be significant flux but poor correlation. This, for instance, is the case in the BOMEX simulation. It is also interesting to note that the upper bound on the top-hat contribution to the variances, for jointly Gaussian processes, is very close to what is found for the top-hat contribution to the $w$ variance for both the BOMEX and the FIRE case when the $E_{i}$ 's are based on the sign of vertical velocity [which ensures $\rho$ $=1$ in Eq. (9)]. This suggests that even though our fields from the LES are not purely Gaussian, the analysis of jointly Gaussian processes does give insight into our results.

For jointly Gaussian processes, any third-order moment vanishes, that is, $\overline{a^{\prime} b^{\prime 2}}=0$. In this case, the tophat model gives exact representation of the third-order moments, because their parameterized values are also zero due to the symmetry of Gaussian PDFs. For nonGaussian processes, $S P V$ may have significant contributions to the total statistics as discussed in the previous simulations.

\section{b. Numerical results from synthetic time series}

To what extent does the upper bound on the contribution of the top-hat contribution to the variance depend on the nature of the signal being analyzed? What is the 

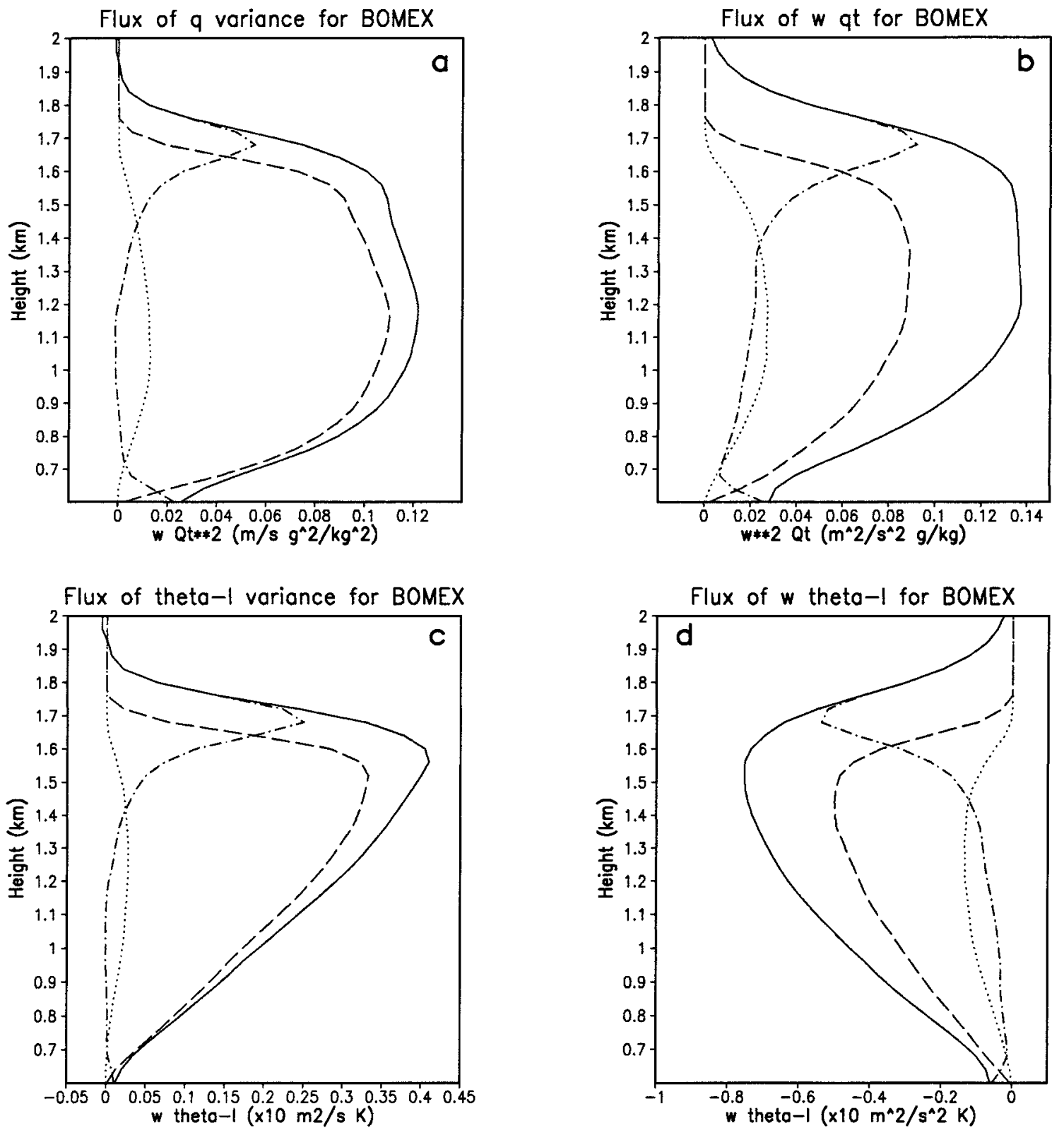

FIG. 10. Contributions of top-hat and subplume variability to the third-order statistics for the BOMEX case. (a) $\overline{w^{\prime} q_{t}^{\prime 2}}$, (b) $\overline{w^{\prime 2} q_{t}^{\prime}}$, (c) $\overline{w^{\prime} \theta_{l}^{\prime 2}}$, and (d) $\overline{w^{\prime 2} \theta_{l}^{\prime}}$. Solid lines represent the total values of the variables; long-dashed, the top-hat contribution from the convective updrafts; dashed-dotted, the contribution from subplume variability in the environment; and dotted, the updraft subplume variability. The environmental top-hat terms are very small and neglected.

physical space interpretation of the magnitude of the SPV terms? To gain insight into these questions we analyze a "toy" time series through the methods already discussed. The time series are built using the bounded cascade model of Cahalan et al. (1994) and Davis et al. (1997) and can be used to generate time series with some nice scaling properties (i.e., we can enforce wavenumber $\kappa$ scaling proportional to $\kappa^{5 / 3}$ over many decades). A further description of how we construct the time series is provided in appendix B.

Here, because we are interested in the upper bound on the top-hat terms we only look at the partitioning of the variance for signals in which the sampling into subsets is based on the sign of the signal itself. We find that for our synthetic time series the top-hat fraction tends to be a strong function of the kurtosis of the signal. This result is illustrated in Fig. 12, where irrespective of the variance contained in a signal, the top-hat fraction appears to be a strictly decreasing function of the kurtosis. Recall that the kurtosis (or flatness) of a signal is simply the ratio of the fourth-order moments to the second-order moments and its value is large when a time 


\section{Flux of q variance for FIRE}

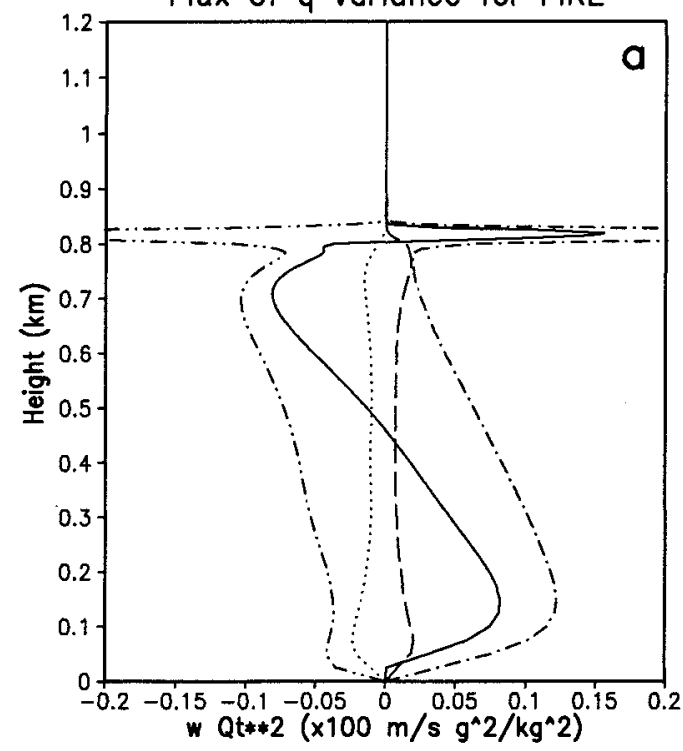

Flux of theta-I variance for FIRE

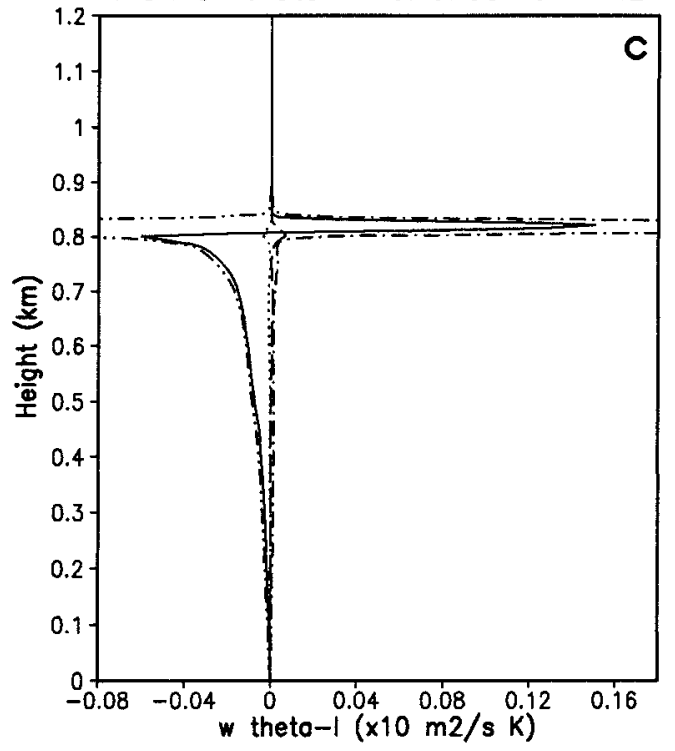

Flux of $w$ gt for FIRE

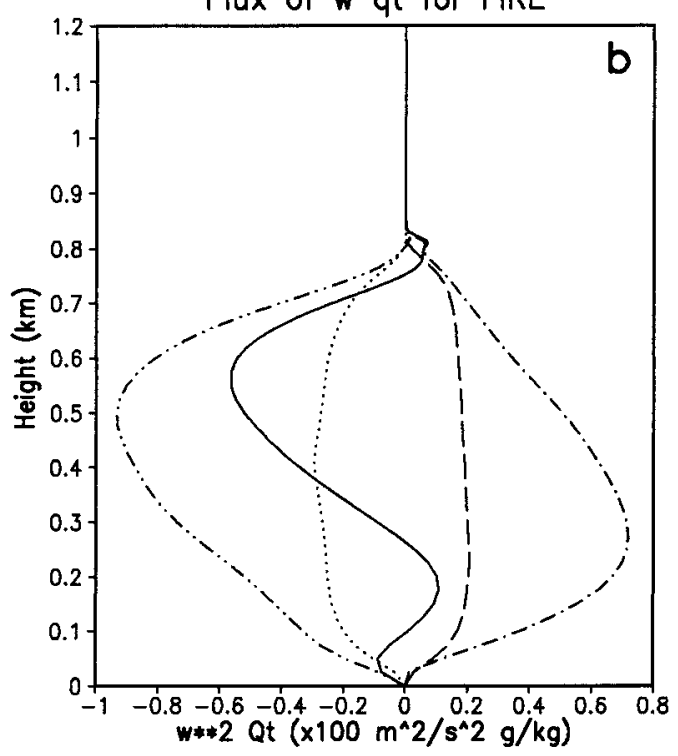

Flux of $w$ theta-1 for FIRE

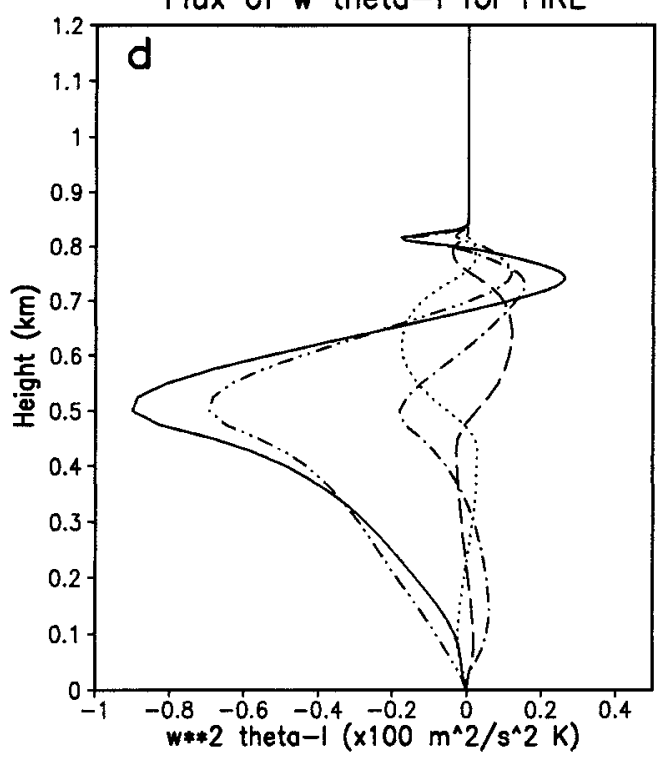

FIG. 11. Contributions of top-hat and subplume variability to the third-order statistics for the FIRE case. (a) $\overline{w^{\prime} q_{t}^{\prime 2}}$, (b) $\overline{w^{\prime 2} q_{t}^{\prime}}$, (c) $\overline{w^{\prime} \theta_{l}^{\prime 2}}$, and (d) $\overline{w^{\prime 2} \theta_{l}^{\prime}}$. Solid lines represent total values of the variables; long-dashed, the top-hat contribution from the updrafts; dotted, the top-hat from the downdrafts; dash-dotted, updraft subplume variability; and dash-dot-dotted, the downdraft subplume variability.

series frequently has extreme values. This result motivated us to look at some simple analytic PDFs, such as uniform, top-hat, triangle, etc. Integration of these distributions further suggests that there is indeed a tendency for the top-hat fraction to increase with decreases in the values of kurtosis as shown in Table 1 . Note that all the PDFs have zero skewness values.

Physically, we can compare time series with smaller and larger kurtosis (e.g., Fig. 13). If we conceptually group the signals into events, each of which has either successive positive or negative points, then each top- hat subset (positive or negative) can be thought of as a composite of all the events (positive or negative) belonging to that subset. Then the $S P V$ can be interpreted as the variability within the individual events as well as among the different events. We find that the difference between a time series with large kurtosis and a time series with a small kurtosis is evident in the amplitude of distinct events. Thus it suggests that the prime contributor to the SPV is the variability among events, rather than variability within events. This view gets further support from the fact that a signal with considerably 

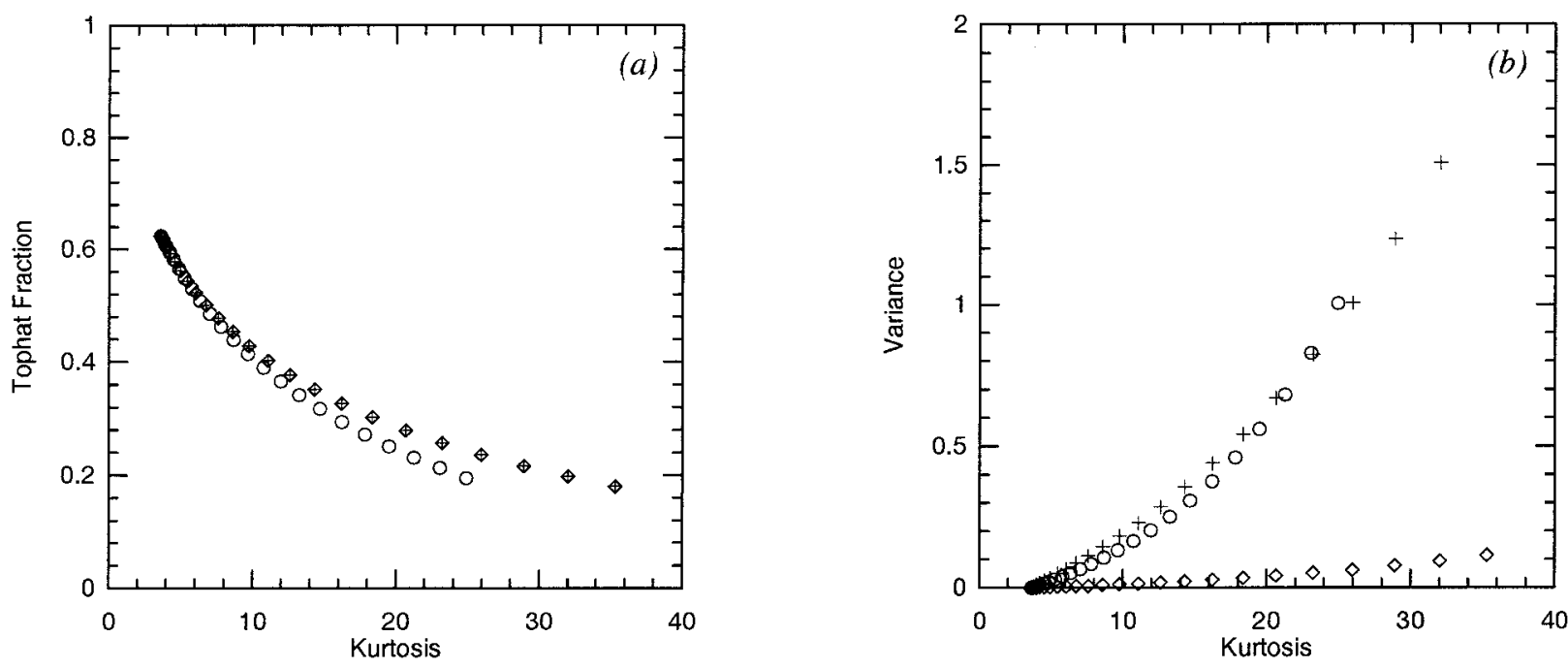

FIG. 12. (a) The top-hat fraction of the variance as a function of kurtosis for fields perfectly correlated with the sampling field. Diamonds are from a time series with relatively small variance, crosses are from a field with relatively large variance. Circles are from time series with relatively large variance but a decade less of an inertial range than the time series corresponding to the crosses. (b) The kurtosis and the variance for the time series analyzed in (a).

less spectral power at small scales tends to not differ substantially in its top-hat fraction from one with more power at small scales (e.g., compare the crosses and circles in Fig. 12, corresponding, respectively, to composites of time series like those in Figs. 13b,c, respectively). This result further justifies the scale truncation implicit in the LES.

\section{Conclusions}

In this work, we have studied the validity of the tophat representation of some second- and third-order turbulence statistics with LES of both a shallow cumulus and a stratocumulus CTBL. The main conclusions of this work are summarized as follows.

The top-hat parameterizations based on the commonly used conditional sampling techniques poorly represent the scalar variance and covariance statistics in these two case simulations, despite the fact that the same parameterization provides realistic or useful estimates for the scalar fluxes. This primarily follows from the two facts. First, most conditional sampling methods are based at least in part on vertical velocity, in which case the variance of a scalar may be large in a region of the flow where it correlates poorly with the velocity. Second, the self-correlation nature of a variance ensures that all the fluctuations contribute to its value, while the top-hat models completely ignore the variability from the top-hat means. For the fluxes, however, the contribution comes mainly from the coherent elements, and the contribution by the subplume variabilities is limited due to their less coherent nature. The analysis of "toy" time series further indicates that variability among individual events within a composite degrades the top-hat representation of the variance more than variability within events.

We also have shown that there is clear structural differences between the BOMEX and FIRE cases in their respective distributions of $w^{\prime}$ and $\varphi^{\prime}$ due to the nature of the different convective circulations. For the BOMEX case, the distribution functions demonstrate apparent bimodal behavior with strong correlation in the convective updrafts and poor correlation in the environment. This phenomenon does not exist in the FIRE case, where the $w^{\prime}-q_{t}^{\prime}$ distribution shows some Gaussian features, while the $w^{\prime}-\theta_{l}^{\prime}$ distribution is basically non-Gaussian due to

TABLE 1. Top-hat fractions for different PDFs. Here, $x$ is a random variable, $b$ a positive parameter, and $\delta$ Dirac delta function.

\begin{tabular}{|c|c|c|c|c|c|c|}
\hline PDF & \multicolumn{2}{|c|}{ Top hat } & Uniform & Triangle & Gaussian & Exponential \\
\hline \multirow{4}{*}{ Expression } & $\delta(x+b)$ & & 1 & $1(, \quad|x|)$ & $\exp \left[-x^{2} /\left(2 b^{2}\right)\right]$ & $1 \quad(|x|)$ \\
\hline & & $x \in(-\infty, 0)$ & $\overline{2 b}$, & $\bar{b}(1-\bar{b})$ & $\sqrt{2 \pi} b$ & $\overline{2 b} \exp \left(-\frac{\bar{b}}{b}\right)$ \\
\hline & $\underline{\delta(x-b)}$ & $x \in(0, \infty)$ & $x \in(-b, b)$ & $x \in(-b, b)$ & $x \in(-\infty, \infty)$ & $x \in(-\infty, \infty)$ \\
\hline & & & 0 , otherwise & 0 , otherwise & & \\
\hline Kurtosis & & 1 & 1.8 & 2.4 & 3 & 6 \\
\hline Top-hat fraction & & 1 & 0.75 & 0.667 & 0.637 & 0.5 \\
\hline
\end{tabular}



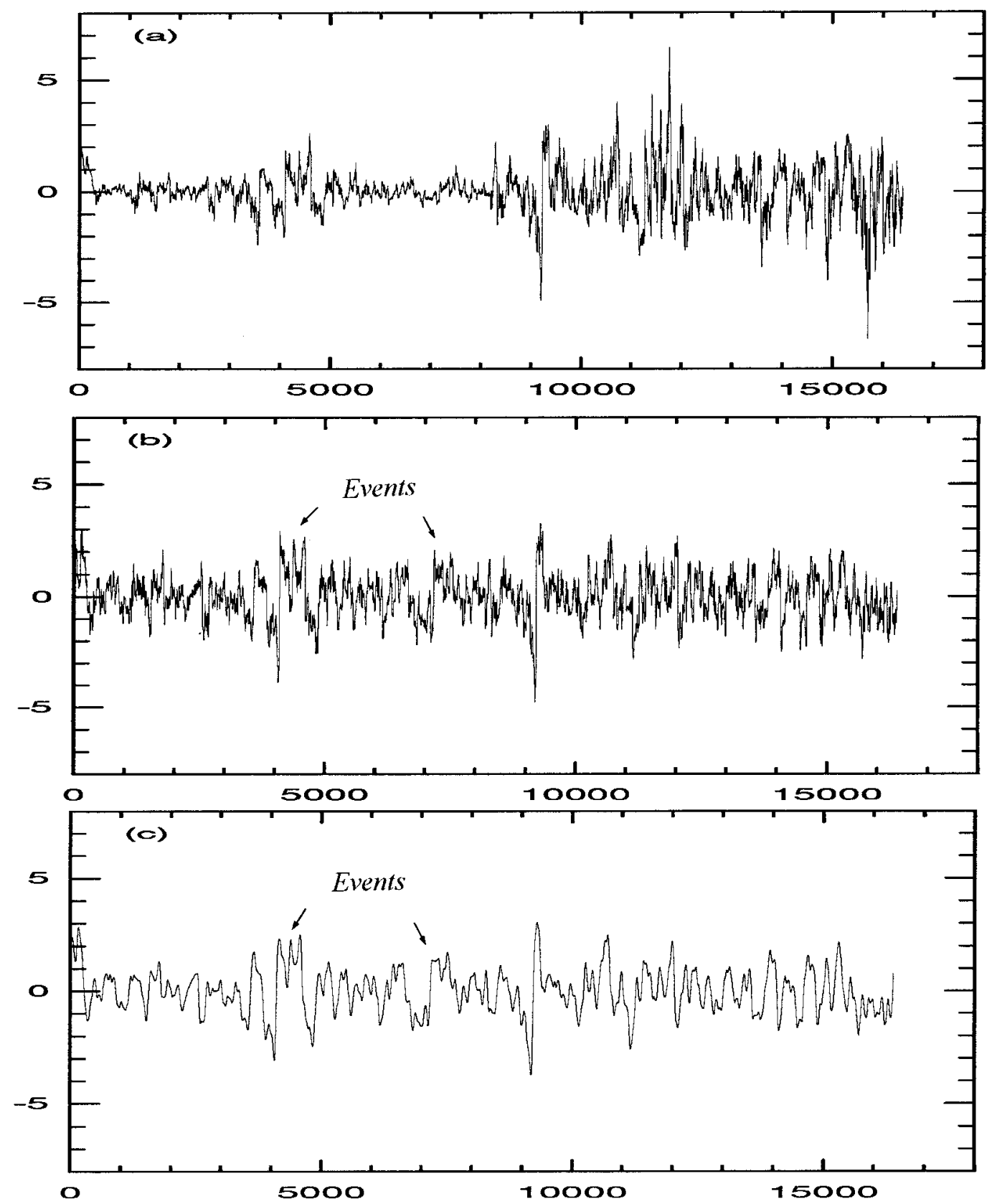

FIG. 13. (a) Time series with large kurtosis. (b) Time series with small kurtosis. (c) Same as (b), but with a decade smaller inertial range. That is, $\kappa_{h}$ in low-pass filter is a factor of 10 smaller.

the influences of the opposing entrainment and radiative processes. This structural difference in part explains why the top-hat models of the second- or third-order scalar fluxes in the cumulus CTBLs are more robust than they are in the stratocumulus CTBLs.

Turbulence in the CTBLs is a manifestation of instability and tends to organize itself in readily identifiable coherent structures. These structures are the agents of the fluxes, and they result from the PBL's attempt to relieve itself of the instability generated by various forc- ings. It is not clear, however, how strongly these forcings are coupled to the non-top-hat parts of the scalar variances. In the BOMEX case, much of the variance occurs in the environment, not in the coherent convective updrafts (Fig. 2). This environmental variance may be explained by decaying structures and may not be as dynamically important as that in the convective updrafts (although it surely affects the evaporation timescale of the detrained inactive clouds so as to influence the cloud cover). In the FIRE case, however, our analysis suggests 
that the variability in the downdrafts be strongly associated with the two dynamic processes, that is, the entrainment and radiative cooling (Fig. 8c). These socalled subplume variabilities can be an important part of the overall CTBL dynamics. For example, Moeng (1986) demonstrated that the buoyancy covariance terms contribute significantly in the flux budget analysis. Therefore, one needs to look at the processes that generate and dissipate the scalar variances and covariances to formulate the best approach to representing them in the context of a particular PBL model.

Although the development of new or improved parameterizations is not a subject of this paper, the results here indeed suggest a number of ways to improve the top-hat parameterizations of the scalar variances. For the cumulus CTBLs, the FDF analysis tends to show two turbulence regimes of the nonlocal cumulus mixing mode and the gradient-dependent quasi-Gaussian mode in the environment. Thus it may be possible to use a turbulence closure model to capture the variance in the environment and top-hat models [Eq. (1)] to simulate the convective updraft's portion. However, to design such a approach, we need to learn more about the nature of the environment turbulence and its relationship to the convective updrafts. As for the stratocumulus CTBLs, one can estimate the SPV terms by assuming a jointly Gaussian distribution as discussed in section 7. A very recent study by Petersen et al. (1999) found that the top-hat parameterizations are significantly improved by relating the SPV terms to the top-hat terms through the empirical correlation factor.

Finally, the conditional sampling technique can be used to perform similar analysis of scalar variance and covariance statistics as is done with the LES calculations in this paper. For example, de Laat and Duynkerke (1998) used the sign of vertical velocity (similar to the conditional sampling used for the FIRE simulation here) to identify updrafts and downdrafts in the aircraft measurement made during the Atlantic Stratocumulus Transition Experiment. One may follow this approach to use in situ measurement to calculate the top-hat and subplume contributions to the scalar variance and the thirdorder statistics to evaluate the validity of (1) and (2). Indeed, such a study is necessary, as modeling studies such as ours predominantly serve the purpose of framing questions and ideas in a way that makes them suitable for testing by observations.

Acknowledgments. We thank Qiang Fu for kindly providing his radiation code, and we appreciate the comments of Andrea Brose, Charlie Cohen, Chin-Hoh Moeng, and David Randall on the original manuscript. Ms. Kate Hinke provided editorial comments. Three anonymous reviewers are thnaked for their thorough reviews. Shouping Wang was supported by NASA/FIRE III and EOS programs. Bjorn Stevens was supported by the Advanced Study Program at NCAR.

\section{APPENDIX A}

\section{Effects of Partial Symmetry of Probability Density Function}

Let us take $q_{t}$ as an example. For the $(m+n)$ th moment $\overline{w^{\prime m} q_{t}^{\prime n}}$, one has

$$
\overline{w^{\prime m} q_{t}^{\prime n}}=\int_{-\infty}^{\infty} w^{\prime m} \int_{-\infty}^{\infty} q_{t}^{\prime n} p\left(w^{\prime}, q_{t}^{\prime}\right) d q_{t}^{\prime} d w^{\prime},
$$

where $m>0$ and $n>0$. If $p\left(w^{\prime}, q_{t}^{\prime}\right) \cong p\left(-w^{\prime}, q_{t}^{\prime}\right)$ for $w^{\prime} \in(-\infty, \infty)$ and $q_{t}^{\prime} \in\left(-\infty, q_{t}^{*}\right)$, where $q_{t}^{*}$ is the cutoff value for the saturated convective updrafts as shown in Fig. 5a, (A1) becomes

$$
\begin{aligned}
\overline{w^{\prime m} q_{t}^{\prime n}}= & \int_{-\infty}^{0} w^{\prime m} \int_{-\infty}^{q_{t}^{*}} q_{t}^{\prime n} p\left(w^{\prime}, q_{t}^{\prime}\right) d q_{t}^{\prime} d w^{\prime} \\
& +\int_{0}^{\infty} w^{\prime m} \int_{-\infty}^{q_{t}^{*}} q_{t}^{\prime n} p\left(w^{\prime}, q_{t}^{\prime}\right) d q_{t}^{\prime} d w^{\prime} \\
& +\int_{-\infty}^{\infty} w^{\prime m} \int_{q_{t}^{*}}^{\infty} q_{t}^{\prime n} p\left(w^{\prime}, q_{t}^{\prime}\right) d q_{t}^{\prime} d w^{\prime} \\
\cong & {\left[1+(-1)^{m}\right] \int_{0}^{\infty} w^{\prime m} \int_{-\infty}^{q_{t}^{*}} q_{t}^{\prime n} p\left(w^{\prime}, q_{t}^{\prime}\right) d q_{t}^{\prime} d w^{\prime} } \\
& +\int_{-\infty}^{\infty} w^{\prime m} \int_{q_{t}^{*}}^{\infty} q_{t}^{\prime n} p\left(w^{\prime}, q_{t}^{\prime}\right) d q_{t}^{\prime} d w^{\prime} . \quad(\mathrm{A} 2)
\end{aligned}
$$

If $m$ is an odd integer, the first integral vanishes and one has

$$
\begin{aligned}
\overline{w^{\prime m} q_{t}^{\prime n}} \cong & \int_{-\infty}^{w^{*}} w^{\prime m} \int_{q_{t}^{*}}^{\infty} q_{t}^{\prime n} p\left(w^{\prime}, q_{t}^{\prime}\right) d q_{t}^{\prime} d w^{\prime} \\
& +\int_{w^{*}}^{\infty} w^{\prime m} \int_{q_{t}^{*}}^{\infty} q_{t}^{\prime n} p\left(w^{\prime}, q_{t}^{\prime}\right) d q_{t}^{\prime} d w^{\prime} .
\end{aligned}
$$

The first integral in the rhs of the above equation should be small with $w^{*}=0$ and $q_{t}^{*}=1.7 \mathrm{~g} \mathrm{~kg}^{-1}$ for the updraft cutoff values in the BOMEX case from Fig. 5, because the integration is over the small sectorlike area enclosed by the minimum-value contour line (in upper left quadrant), $w^{\prime}=0$ and $q_{t}^{\prime}=1.7 \mathrm{~g} \mathrm{~kg}^{-1}$. Thus one has

$$
\overline{w^{\prime m} q_{t}^{\prime n}} \cong \int_{w^{*}}^{\infty} w^{\prime m} \int_{q_{t}^{*}}^{\infty} q_{t}^{\prime n} p\left(w^{\prime}, q_{t}^{\prime}\right) d q_{t}^{\prime} d w^{\prime} .
$$

The above equation suggests that the total moment can be approximated by the contribution only from the updrafts. The same conclusion can be reached for the moment with an $n$ being an odd number and a probability density function that is approximately symmetric about $q_{t}^{\prime}=0$. One can apply a similar procedure to $\theta_{l}$ to obtain the same conclusion. 


\section{APPENDIX B}

\section{Time Series Model}

The model we use to generate simple time series with nice scaling properties is the bounded cascade model of Cahalan et al. (1994). This model is thoroughly described by the National Aeronautics and Space Administration group (see, for instance, Marschak et al. 1994; Davis et al. 1997). The purpose of this appendix is to summarize its features and discuss the details of its implementation for our study.

Mathematically it can be formulated as follows: Let $f_{j}(x) 0 \leq x \leq L$ be piecewise constant on $2 j$ intervals, each of length $r^{j}=L / 2^{j}$. Then

$$
f_{j+1}(x)=W_{j+1}(x ; p, H) f_{j}(x),
$$

where

$$
W_{j+1}(x ; p, H)= \begin{cases}{[1+\Xi(k)(1-2 p)] r_{j}^{H}} & x \in\left(k r_{j},(k+1 / 2) r_{j}\right) \\ {[1-\Xi(k)(1-2 p)] r_{j}^{H}} & x \in\left((k+1 / 2) r_{j},(k+1) r_{j}\right),\end{cases}
$$

where $k=0,1, \ldots, 2^{j}-1$ indexes $W$ over the $2^{j}$ intervals along $L ; \Xi(k)= \pm 1$ with the sign chosen randomly for each $k$ (that is, for each interval spanning $L) ; p \in[0,1 / 2]$; and $H$ is a real number greater than zero.

The model [(B.1) and (B.2)] generates arbitrarily long sequences of numbers (which we hereafter refer to as time series) with nice scaling properties. Physically it can be interpreted as taking intervals with constant mass, bisecting them, and redistributing mass between the bisected components in a way that depends on parameters $p$ and $H$. The procedure is defined recursively, so that it generates a $2^{j}$-point time series after $j$ applications. It preserves the first integral of the time series, namely, the mean value such that $\overline{f_{j}(x)}=f_{0}(x)$. For a given $H, p$ controls the amount of mass redistributed between intervals; $H$ controls how this fraction changes with the level of recursion. As $j$ becomes large the power spectrum of the sequence scales with $\kappa^{-\beta}$, where $\beta$ is determined by $H$ such that $1<\beta(H)=\min (2 H, 1)+$ $1<2$, and $\kappa$ is the wavenumber (Davis et al. 1997).

In our analysis we generate two independent time series (where independence is obtained by starting the procedure using a different seed in our random number generator) with $j=14$ and then take their difference. This procedure minimizes the skewness. We next filter large and small scales from the time series by multiplying the Fourier amplitudes by

$$
\begin{aligned}
{\left[1+\left(\kappa / \kappa_{l}\right)^{2}+\right.} & \left.0.5\left(\kappa / \kappa_{l}\right)^{3}+0.1\left(\kappa / \kappa_{l}\right)^{4}\right]^{-1} \\
& \text { for } \kappa \geq \kappa_{l}
\end{aligned}
$$

and by

$$
\begin{gathered}
{\left[1+\left(\kappa_{H} / \kappa\right)^{2}+0.5\left(\kappa_{H} / \kappa\right)^{3}+0.1\left(\kappa_{H} / \kappa\right)^{4}\right]^{-1}} \\
\text { for } \kappa \leq \kappa_{H} .
\end{gathered}
$$

By building time series from signals with larger or smaller average masses it is possible to modulate the variance of the difference time series, without significantly affecting other low-order statistics. For most of our analysis we average our results from 100 independent time series. Typically we consider time series generated with $H=1 / 3$ fixed so as to generate time series that scale over several decades with $\kappa^{-5 / 3}$. The behavior of the top-hat partitioning of the variance is then considered as a function of $p$.

\section{REFERENCES}

Abdella, K., and N. McFarlane, 1997: A new second-order turbulence closure scheme for the planetary boundary layer. J. Atmos. Sci., 54, 1850-1867.

Albrecht, B. A., 1981: Parameterization of trade-cumulus cloud amounts. J. Atmos. Sci., 38, 97-105.

- M. P. Jensen, and W. J. Syrett, 1995: Marine boundary layer structure and fractional cloudiness. J. Geophys. Res., 100, 14 209-14 222.

Arakawa, A., and W. H. Schubert, 1974: Interaction of a cumulus cloud ensemble with the large scale environment: Part I. J. Atmos. Sci., 31, 674-701.

Betts, A. K., 1973: Non-precipitating cumulus convection and its parameterization. Quart. J. Roy. Meteor. Soc., 99, 178-196.

Bretherton, C. S., and Coauthors, 1999: An intercomparison of radiatively-driven entrainment and turbulence in a smoke cloud, as simulated by different numerical models. Quart. J. Roy. Meteor. Soc., 125, 391-423.

Cahalan, R. F., W. Ridgway, W. J. Wiscombe, and T. L. Bell, 1994: The albedo of fractal stratocumulus clouds. J. Atmos. Sci., 51, 2434-2455.

Davis, A., A. Marshak, R. Cahalan, and W. Wiscombe, 1997: The Landsat scale break in stratocumulus as a three-dimensional radiative transfer effect: Implications for cloud remote sensing. $J$. Atmos. Sci., 54, 241-260.

de Laat, A. T., and P. Duynkerke, 1998: Analysis of ASTEX-stratocumulus data using a mass-flux approach. Bound.-Layer Meteor., 86, 63-87.

Fu, Q., and K. N. Liou, 1992: On the correlated k-distribution method for radiative transfer in nonhomogeneous atmospheres. J. Atmos. Sci., 49, 2139-2156.

— and convection in simulated tropical cloud clusters. J. Atmos. Sci., 52, 1310-1328.

Marshak, A., A. Davis, R. F. Cahalan, and W. J. Wiscombe, 1994: Bounded cascade models as non-stationary multifractals. Phys. Rev, E49, 55-79.

Moeng, C.-H., 1986: Large-eddy simulation of a stratus-topped 
boundary layer. Part I: Structure and budgets. J. Atmos. Sci., 43, 2886-2900.

- and Coauthors, 1996: Simulation of a stratocumulus topped planetary boundary layer: Intercomparison among different numerical codes. Bull. Amer. Meteor. Soc., 77, 261-278.

Penc, R. S., and B. A. Albrecht, 1986: Parametric representation of heat and moisture fluxes in cloud-topped mixed layers. Bound.Layer Meteor., 38, 225-248.

Petersen, A. C., C. Beets, H. V. Dop, P. Duynkerke, and A. P. Siebesma, 1999: Mass-flux characteristics of reactive scalars in the convective boundary layer. J. Atmos. Sci., 56, 37-56.

Randall, D. A., Q. Shao, and C.-H. Moeng, 1992: A second-order bulk boundary layer-model. J. Atmos. Sci., 49, 1903-1923.

Schumann, U., and C.-H. Moeng, 1991: Plume fluxes in clear and cloudy convective boundary layers. J. Atmos. Sci., 48, 17461757.

Siebesma, A. P., 1996: On the mass flux approach for atmospheric convection. ECMWF Seminar Proc. New Insights and Approaches to Convective Parameterization, Reading, United Kingdom, ECMWF, 25-57.
—, and J. W. M. Cuijpers, 1995: Evaluation of parametric assumptions for shallow cumulus convection. J. Atmos. Sci., 52, $650-666$.

Sommeria, G., and J. W. Deardorff, 1977: Subgrid-scale condensation models of nonprecipitating clouds. J. Atmos. Sci., 34, 345-355.

Stevens, B., G. Feingold, W. R. Cotton, and R. L. Walko, 1996: Elements of the microphysical structure of numerically simulated nonprecipitating stratocumulus. J. Atmos. Sci., 53, 980-1006.

— C.-H. Moeng, and P. P. Sullivan, 1999: Large-eddy simulations of radiatively driven convection: Sensitivities to the representation of small scales. J. Atmos. Sci., 56, 3963-3984.

Wang, S., and B. A. Albrecht, 1986: A stratocumulus model with an internal circulation. J. Atmos. Sci., 43, 2373-2391.

Wyngaard, J. C., and C. H. Moeng, 1992: Parameterizing turbulent diffusion through the joint probability density. Bound.-Layer Meteor., 60, 1-13.

Young, G. S., 1988: Turbulence structure in the convective boundary layer. Part II: Phoenix 78 Aircraft observations of thermals and their environment. J. Atmos. Sci., 45, 727-735.

Zalesak, S. T., 1979: Fully multidimensional flux-corrected transport algorithms for fluids. J. Comput. Phys., 11, 335-362. 